\title{
Improved off-shell scattering amplitudes in string field theory and new computational methods
}

\author{
Itzhak Bars \\ Department of Physics, USC, Los Angeles, California 90089-0484, USA \\ and Theory Division, CERN, CH-1211 Geneva 23, Switzerland \\ I. Y. Park \\ Department of Physics, The Ohio State University, Columbus, Ohio 43210, USA
}

(Received 9 December 2003; published 27 April 2004)

\begin{abstract}
We report on new results in Witten's cubic string field theory for the off-shell factor in the 4-tachyon amplitude that was not fully obtained explicitly before. This is achieved by completing the derivation of the Veneziano formula in the Moyal star formulation of Witten's string field theory (MSFT). We also demonstrate a detailed agreement of MSFT with a number of on-shell and off-shell computations in other approaches to Witten's string field theory. We extend the techniques of computation in MSFT, and show that the $j=0$ representation of $\operatorname{SL}(2, R)$ generated by the Virasoro operators $L_{0}, L_{ \pm 1}$ is a key structure in practical computations for generating numbers. We provide more insight into the Moyal structure that simplifies string field theory, and develop techniques that could be applied more generally, including nonperturbative processes.
\end{abstract}

DOI: 10.1103/PhysRevD.69.086007

PACS number(s): 11.25.Sq

\section{INTRODUCTION}

In this paper we will report on new results in Witten's string field theory [1] for the off-shell 4-tachyon amplitude that was not fully computed explicitly before. In previous computations the off-shell factor $f(x)$ was obtained in an implicit form [2,3], while an explicit computation reported only the first two terms in an expansion in the vicinity of the integration limit of the parameter $x$ [3]. These results were obtained by using mainly conformal mapping techniques [4] and followed the methods of Giddings' original computation [5] of the on-shell Veneziano amplitude, which could be extended to off-shell under the guidance of the oscillator formulation of string field theory [6-8]. In this paper we will obtain a fairly comprehensive profile of the off-shell factor $f(x)$ in its entire range $0 \leqslant x \leqslant 1$ by giving the explicit form to tenth order in the parameter $x$ (first two terms in agreement with [3]), obtaining a plot in the full range, and computing the critical slope at a turning point in midrange that determines differentiability. Contrary to our result, previously it was thought that the function $f(x)$ was not differentiable at $x=1 / 2$, where it was not well understood. These are achieved in the Moyal star formulation of Witten's string field theory (MSFT) [9-15].

The usual approach of computation starts with a precise formulation of Witten's string field theory, such as the oscillator formulation, to derive a formal expression for a string Feynman diagram in terms of the cubic vertex defined in terms of the Neumann coefficients. After this step a jump is made to conformal maps from an analog model [4] and the real computation is performed by using conformal field theory, if the conformal map can be constructed. The desired conformal maps can be found explicitly only in certain lucky cases, and the four point function is one of them. The conformal map procedure has been used virtually in every successful analytic computation, while the oscillator basis is directly pursued mainly with numerical studies using level truncation $[18,19]$ because of the complexity of the Neu- mann coefficients. These have been some of the challenging features of Witten's string field theory in various formulations $[7,20]$ that, despite the beauty of Witten's basic action, have led to limited results in string field theory.

Some of the complexities of other approaches are not present, or take an easier form in MSFT. In this formulation the string joining star product is the simple Moyal product, and this reproduces directly results in string field theory, in agreement with conformal field theory, but without mapping back to conformal field theory or other intermediate steps. Therefore some of the lingering problems in string field theory seem to be good testing grounds for MSFT.

In this paper we apply the MSFT techniques to the offshell 3-point and 4-point amplitudes. We will derive the Veneziano amplitude directly from MSFT and determine the offshell factor far more accurately compared to previous computations. In this process we demonstrate that MSFT agrees in detail with other approaches while bringing efficiency into the computations, and also obtain new results in MSFT that other approaches could test. We view these computations as a preparation for our ultimate aim which is the investigation of nonperturbative string theory phenomena by using the simpler MSFT. We believe the techniques and insights developed in this paper will be useful for this purpose. In fact we find that some of the new results and insight gained by this work impact the way to compute nonpertubative quantities in string field theory as discussed in Sec. VI.

In the rest of this section we will describe briefly MSFT and introduce some notation. In Sec. II we discuss off-shell 3 -point functions. This is necessary to understand the behavior of the theory with respect to a cutoff in mode space, and to define the physical string coupling as opposed to the bare divergent coupling that appears in the action. The results we obtain for the off-shell 3-point amplitudes demonstrate detailed agreement between MSFT and conformal field theory or the oscillator formulation of string field theory.

In Sec. III we analyze the off-shell 4-tachyon amplitude. We show directly from MSFT, without connecting through 
conformal field theory, that on-shell we obtain the Veneziano amplitude. Furthermore we obtain the off-shell factor $f(x)$ and compute the first ten terms in an expansion in powers of $x$, provide a plot in its full range $0 \leqslant x \leqslant 1$, and determine its differentiability at a turning point. Previously it was thought that the derivative was discontinuous at midrange and only the lowest two terms were explicitly computed [3] (in agreement with our result). Our computation provides the most comprehensive information on the off-shell 4-tachyon amplitude produced so far in string field theory.

In Sec. IV we develop the details of the tools that allowed us to perform the computations in Secs. II and III and which would be applicable more generally to other computations.

In Sec. V we discuss the SL $(2, R)$ generated by the Virasoro generators $L_{0}, L_{ \pm 1}$, whose $j(j+1)=0$ representation deeply underlies the structures that appear in our computations. By using some group theoretic properties of this very special representation of $\operatorname{SL}(2, R)$ we develop tools for computation in string field theory that are needed in our paper to generate numbers. We also compare the discrete and continuous Moyal bases which are simply two different bases of the $j=0$ representation.

In Sec. VI we discuss some of the impact that our present computations have on the nonperturbative landscape, and then conclude in Sec. VII. In the Appendix we give further results produced through the techniques in Sec. IV, and relations to Neumann coefficients.

The action of Witten's cubic string field theory [1] in the MSFT formalism in the Siegel gauge is

$S(A)=-\int d^{d} \bar{x} \operatorname{Tr}\left(\frac{1}{2 \alpha^{\prime}} A \star\left(L_{0}-1\right) A+\frac{g_{0}}{3} A \star A \star A\right)$,

where $L_{0}$ is given in Eqs. (6.5), (6.6). The zero mode ghosts have already been dealt with [15] so they no longer appear. Hence the field $A$ here is equivalent to the physical field in Witten's theory. The string field $A\left(\bar{x}, x_{e}, p_{e}\right)$ is written in a mixed position-momentum basis, which is equivalent to a Fourier transform of the purely position basis. In this basis string joining is represented by the usual Moyal star product [9] in a noncommutative phase space $\left(x_{e}, p_{e}\right)$ labeled by the even modes $e=2,4,6, \ldots,{ }^{1}$ The star product $\star$ is local in

\footnotetext{
${ }^{1}$ The position basis is given in terms of the even and odd modes $\left(x_{e}, x_{o}\right)$. The Fourier transform in the odd modes maps to the space $\left(x_{e}, p_{o}\right)$. Witten's string joining star product becomes a nondiagonal Moyal product in the space $\left(x_{e}, p_{o}\right)$ [9]. This is diagonalized by defining $p_{e}$ as an infinite combination of the $p_{o}=p_{e} T_{e o}$ by introducing the special matrix $T_{e o}$ given in Eq. (4.4). In this way we arrive to the noncommutative space $\left(x_{e}, p_{e}\right)$ with a diagonal Moyal product whose meaning is string joining. We emphasize that this $p_{e}$ is a definition in terms of $p_{o}$, it should not be confused with the first quantized momentum that is canonical to $x_{e}$, whose representation in this space is the derivative $i \partial_{x_{e}}$. However, interestingly this can be reproduced in the noncommutative geometry (string joining) relation $-i \partial_{x_{e}} A\left(\bar{x}, x_{e}, p_{e}\right)=\left[p_{e}, A\left(\bar{x}, x_{e}, p_{e}\right)\right]_{\star}$. A closely related continuous Moyal basis [16] is obtained by orthogonality transformations, as will be discussed later in the paper.
}

the midpoint variable $\bar{x}$, and is independent for each $e$. This separation of variables and the simplicity of the Moyal star are the main conceptual and practical simplifications that lead to new progress by overcoming midpoint problems in other approaches and opening up easier computational techniques in MSFT.

It has been shown in [9-15] through some explicit computations that MSFT is in full agreement with other computational approaches to Witten's string field theory, including the oscillator formulation and conformal field theory [7-20]. In particular, the Moyal star reproduces the Neumann coefficients that define the vertices in the oscillator formulation of string field theory $[11,15]$. Furthermore, the MSFT propagator has the usual free string spectrum. Therefore, even though it is a very different computational formalism, due to the one to one correspondence described in [13] we expect identical final results between MSFT and oscillator approach computations of any string Feynman graph. This expectation will be confirmed in detail in this paper. This demonstrates once again, and in greater detail, that MSFT is a precise representation of Witten's string field theory.

The string in 26 dimensions is supplemented with two additional fermionic dimensions that describe the conformal ghosts $b, c$, with the appropriate generalization of the Moyal star product for fermions. The traditional perturbative string states (tachyon, vector, etc.) are identified through the usual expansion

$$
\begin{aligned}
A\left(x_{c m}, x_{e}, p_{e}\right)= & T\left(x_{c m}\right) A_{0}\left(x_{e}, p_{e}\right) \\
& +V_{\mu}\left(x_{c m}\right)\left(\alpha_{-1}^{\mu} A_{0}\left(x_{e}, p_{e}\right)\right)+\cdots
\end{aligned}
$$

where $A_{0}\left(x_{e}, p_{e}\right)$ is the perturbative vacuum string field configuration and $\alpha_{-n}^{\mu}$ is a differential operator representation of string oscillators in the space $\left(x_{e}, p_{e}\right)[11,15] . A_{0}$ is a specific normalized Gaussian $\operatorname{Tr}\left(A_{0} \star A_{0}\right)=1$, that represents the vacuum $L_{0} A_{0}=0$, including ghosts [15] (see also [17]). It is given by

$$
\begin{aligned}
A_{0}(\xi)= & \left|\operatorname{det} 4 m_{0}\right|^{d / 4}\left|\operatorname{det} 4 m_{0}^{-1}\right|^{-2 / 4} \exp \left(-\eta_{\mu \nu} \xi^{\mu} m_{0} \sigma^{-1} \xi^{\nu}\right. \\
& \left.+i \varepsilon_{m n} \xi^{m} m_{0}^{-1} \sigma^{-1} \xi^{n}\right), \quad d=26,
\end{aligned}
$$

where $\xi^{\mu}, \xi^{m}$ are the noncommutative coordinates written as a doublet for each $e$, with the bosonic part $\xi_{i}^{\mu}=\left(x_{e}^{\mu}, p_{e}^{\mu}\right)$ for matter and fermionic part $\xi^{1}=\left(x_{e}^{b} / \sqrt{2 \alpha^{\prime}},-\sqrt{2 \alpha^{\prime}} p_{e}^{c}\right), \xi^{2}$ $=\left(x_{e}^{c} / \sqrt{2 \alpha^{\prime}}, \sqrt{2 \alpha^{\prime}} p_{e}^{b}\right)$ for the $b, c$ ghosts. ${ }^{2}$ Each pair $(x, p)$ satisfies standard commutation-anticommutation rules under the star product. These can be written compactly as $\left[\xi_{i}^{\mu}, \xi_{j}^{\nu}\right]_{\star}=\eta^{\mu \nu} \sigma_{i j}$ for matter and $\left\{\xi_{i}^{m}, \xi_{j}^{n}\right\}_{\star}=-i \varepsilon^{m n} \sigma_{i j}$ for ghosts, where the symbol $\sigma=-\theta \sigma_{2}$ is the Pauli matrix $\sigma_{2}$ in

\footnotetext{
${ }^{2}$ Relative to $[14,15]$ we are improving notation by introducing $\xi^{m}$, with $m=1,2$, and the $\operatorname{Sp}(2)$ metric $\varepsilon_{m n}$, for the sake of connecting with an upcoming paper in which we will discuss some useful hidden symmetries that connect matter and ghosts in the MSFT formalism, and further simplify the structure and computations.
} 
the doublet space multiplied with a noncommutativity parameter $\theta$. We take $\theta=1$ by a choice of units. The $m_{0}$ that appears in the vacuum state $A_{0}(\xi)$ is a matrix in mode space determined by $L_{0} A_{0}=0$ as shown in [11]. Although $m_{0}$ is a simple matrix, we will not need it explicitly in this paper.

The perturbative particle fields $T\left(x_{c m}\right), V_{\mu}\left(x_{c m}\right)$, etc. are expressed as functions of the center of mass $x_{c m}$. The star product $\star$ is local in the midpoint of the string $\bar{x}$, not in the center of mass $x_{c m}$. Therefore, before evaluating the interaction for any perturbative field $T\left(x_{c m}\right), V_{\mu}\left(x_{c m}\right)$, etc. one must first write the center of mass in terms of the midpoint $x_{c m}=\bar{x}+w_{e} x_{e}$, where $w_{e}=-\sqrt{2}(-1)^{e / 2}$. This is a crucial step in computations of the star products. The midpoint had been a source of numerous problems in the split string formalism, and the resolution first was given in the context of MSFT in [11] with the simple prescription just described.

In the quadratic term the star product plays no role, and could be removed, as is usual for the Moyal product. The coefficient of the quadratic term in the action is chosen such that the particle fields are correctly normalized (after taking into account the definitions of the trace $\mathrm{Tr}$ including ghosts and the Virasoro operator $L_{0}$ as given in [15])

$$
\begin{aligned}
S_{\text {quadratic }}= & -\int d^{d} \bar{x}\left(\frac{1}{2} \partial_{\mu} T \partial^{\mu} T+\frac{1}{4} F_{\mu \nu} F^{\mu \nu}+\ldots\right) \\
= & \int d^{d} \frac{1}{x} \frac{1}{2}\left(\partial_{0} T \partial_{0} T-\vec{\nabla} T \cdot \vec{\nabla} T\right)+\frac{1}{2} \partial_{0} V^{\mu} \partial_{0} V_{\mu} \\
& +\cdots .
\end{aligned}
$$

Then from the cubic term one finds the Feynman rules and computes the amplitudes as described in $[13,15]$. For each external line we insert a string field representative of a particle. In particular an incoming tachyon with momentum $k^{\mu}$ is represented by the string field $A_{0}\left(x_{e}, p_{e}\right) e^{i k \cdot x_{c m}}$, where $A_{0}$ is the normalized vacuum field given above, and $e^{i k \cdot x_{c m}}$ is the center of mass plane wave which is part of $T\left(x_{c m}\right)$. To compute its interactions one must write it in the form $A_{0}\left(x_{e}, p_{e}\right) e^{i k \cdot\left(\bar{x}+w_{e} x_{e}\right)}$ which is a shifted Gaussian in $\left(x_{e}, p_{e}\right)$ space. These details are fully explained in [9-15], where it is also shown how to compute the star product and the trace with efficient methods based on a monoid algebra of shifted Gaussians [11].

In the expressions below the constant matrix $t_{e o}$ and vectors $w_{e}, v_{o}$ in even or odd mode space (and related matrices $\left.T_{e o}, R_{o e}\right)$ are fundamental matrices in MSFT that encode the joining of strings [9-16], and are given explicitly in Eqs. (4.3), (4.4). The matrices $\kappa_{o}, \kappa_{e}$ are diagonal matrices that represent the odd or even oscillator frequencies $\kappa_{o}$ $=\operatorname{diag}(o), o=1,3,5, \ldots$ and $\kappa_{e}=\operatorname{diag}(e), e=2,4,6, \ldots$ as in Eq. (3.6) below. A bar on top of a square or column matrix symbol, such as $\bar{t}, \bar{v}$, etc. means the transpose of the matrix. In certain computations, to avoid associativity anomalies these infinite matrices must be replaced with their regulated $N \times N$ version as given in [10,13-15] and then $N$ must be sent to infinity at the end. The regulated matrices obey some nice algebraic properties which are also shared by the infinite matrices, thereby permitting analytic computation in the finite $N$ version. A particular form of the regulator which we have found useful in some computations is given in footnote 6 .

It is necessary to use the regulator in those computations where we suspect anomalous behavior, but otherwise the unregulated matrices can be used, as we will do for most of the computations in this paper. It is interesting to note that the regulated matrices with only a few modes ( $\operatorname{small} N$ ) reproduce approximately most of the numerical results we obtain with more sophisticated methods at $N=\infty$. An example is the Neumann matrices as given in the appendix of [15], and many of the numbers computed in the current paper, although we do not make the effort to demonstrate this point in this paper.

\section{OFF-SHELL 3-POINT FUNCTIONS}

In this section first we briefly outline the off-shell 3-point functions to establish the relation between the bare coupling $g_{0}$ that appears in the action of MSFT and the on-shell tachyon coupling $g$, which is identified with the string coupling. The relation between the two involves a factor which diverges with the number of modes $2 N$ as $g_{0} \sim(2 N)^{3 / 2} g$ as will be explained later. All other scattering amplitudes are proportional to some power of the bare coupling $g_{0}$, and this must be first written in terms of the finite on-shell tachyon coupling $g$. After this step, it is seen that all amplitudes are finite. In this process one finds a renormalization factor in front of the amplitudes multiplying a power of the on-shell coupling $g$. An example of this is the factor $g^{2}(27 / 16)^{3} / 4$ for the 4-tachyon amplitude in Eq. (3.5) below.

The off-shell 3-tachyon amplitude is obtained by using the Feynman rules (note $\frac{1}{3} g_{0} \times 3 !=2 g_{0}$ ) and inserting the tachyon field for the external leg in the Feynman graph, leading to the expression

$$
\begin{aligned}
g_{123}\left(k_{i}\right)= & 2 g_{0} \int d^{d} \bar{x} \operatorname{Tr}\left(A_{0} e^{i k_{1} \cdot\left(\bar{x}+w x_{e}\right)}\right. \\
& \left.\star A_{0} e^{i k_{2} \cdot\left(\bar{x}+w x_{e}\right)} \star A_{0} e^{i k_{3} \cdot\left(\bar{x}+w x_{e}\right)}\right) .
\end{aligned}
$$

The $\bar{x}$ integral gives a momentum conservation delta function $(2 \pi)^{26} \delta^{(26)}\left(k_{1}+k_{2}+k_{3}\right)$, while the trace and star products are easily evaluated by using the Bose-Fermi monoid rules developed in $[11,15]$. The result is

$$
g_{123}\left(k_{i}\right)=g\left(\frac{27}{16}\right)^{3 / 2-(1 / 2) \alpha^{\prime}\left(k_{1}^{2}+k_{2}^{2}+k_{3}^{2}\right)},
$$

multiplied with the delta function. This is in full agreement with previous studies of the off-shell 3-tachyon amplitude $[6,7,2]$. It gives precisely $g$ on shell by definition $\left.g_{123}\left(k_{i}\right)\right|_{\alpha^{\prime} k_{i}^{2}=1}=g$. The remaining off-shell factor in our calculation initially has the form $\exp \left(\omega\left[\frac{3}{2}-\frac{1}{2} \alpha^{\prime}\left(k_{1}^{2}+k_{2}^{2}\right.\right.\right.$ $\left.\left.\left.+k_{3}^{2}\right)\right]\right)$, with $\omega=\left(1 / 2 \alpha^{\prime}\right)(w, 0) \sigma m_{0}\left(3+m_{0}^{2}\right)^{-1}(w, 0)^{T}$. This is obtained easily by evaluating the star products and trace in Eq. (2.1) by using the simple monoid methods in $[11,15]$. 
Using the definition of $m_{0}$ this simplifies to $\omega=2 \bar{v} \kappa_{o}^{-1 / 2}(3$ $+\bar{t} t)^{-1} \kappa_{o}^{-1 / 2} v$, which is then evaluated as $\omega=\ln (27 / 16)$ later in this paper in Eqs. (4.13), (4.20), to produce the result in Eq. (2.2). Similarly, in the same computation, the relation between the on-shell $g$ and the bare coupling $g_{0}$ initially takes the following form $g_{0}=\frac{1}{2} g\left(\frac{27}{16}\right)^{3 / 2} \operatorname{det}((3$ $\left.\left.+m_{0}^{2}\right) / 4\right)^{d / 2} \operatorname{det}\left(\left(3+m_{0}^{-2}\right) / 4\right)^{-2 / 2}\left|\operatorname{det} m_{0}\right|^{-d / 4}\left|\operatorname{det} m_{0}^{-1}\right|^{-2 / 4}$. After inserting $m_{0}$ it becomes

$$
\begin{aligned}
g_{0}= & \frac{1}{2} g\left(\frac{27}{16}\right)^{3 / 2}(\operatorname{det}(t \bar{t}))^{-(d-6) / 4} \operatorname{det}\left(\frac{3+(t \bar{t})}{4}\right)^{d} \\
& \times \operatorname{det}\left(\frac{1+3(t \bar{t})}{4}\right)^{-2}, \quad d=26 .
\end{aligned}
$$

The right hand side of Eq. (2.3) is divergent as $(2 N)^{(d-6) / 8-d / 18+4 / 9}$ which becomes $(2 N)^{3 / 2}$ for $d=26$ as will be later shown in Eq. (4.32). However this divergence is everywhere reabsorbed into the definition of the on shell $g$ just as in the 3-tachyon case. After this step, there still remains similar determinants that individually produce a divergence or zeros at large $N$, but they combine together to give finite answers magically as long as $d=26$. We will see an example of this impressive fact below in the 4-tachyon amplitude [see the determinants in Eq. (3.5) and the computation in Eqs. (4.24)].

A similar computation can be performed for the vector particle for an incoming wave with momentum $k^{\mu}$, which is represented by the string field

$$
A_{V}\left(x_{c m}, x_{e}, p_{e}, k\right)=e^{i k \cdot x_{c m}} A_{0}\left(x_{e}, p_{e}\right) \varepsilon_{\mu}(k)\left(p^{\mu} T\right)_{1} \sqrt{2 \alpha^{\prime}},
$$

where $\varepsilon_{\mu}(k)$ is the polarization and the last factor $p_{e}^{\mu} T_{e 1} \sqrt{2 \alpha^{\prime}}$ resulted from applying the oscillator $[11,15]$ $\alpha_{-1}^{\mu} A_{0}\left(x_{e}, p_{e}\right)$. For example, the coupling between the vector and two tachyons is (omitting the Chan-Paton factors at the string ends)

$$
\begin{aligned}
g_{12 V_{3}}\left(k_{i}\right)= & 2 g_{0} \int d^{d} \bar{x} \operatorname{Tr}\left(A_{0} e^{i k_{1} \cdot\left(\bar{x}+w x_{e}\right)} \star A_{0} e^{i k_{2} \cdot\left(\bar{x}+w x_{e}\right)}\right. \\
& \left.\star A_{0} e^{i k_{3} \cdot\left(\bar{x}+w x_{e}\right)} \varepsilon_{\mu}\left(k_{3}\right)\left(p^{\mu} T\right)_{1} \sqrt{2 \alpha^{\prime}}\right)
\end{aligned}
$$

Following the monoid methods in $[11,15]$ that led to Eq. (2.2) this is evaluated in almost the same way, giving

$$
g_{12 V_{3}}\left(k_{i}\right)=\frac{1}{2} g\left(k_{1}-k_{2}\right) \cdot \varepsilon\left(k_{3}\right)\left(\frac{27}{16}\right)^{1-(1 / 2) \alpha^{\prime}\left(k_{1}^{2}+k_{2}^{2}+k_{3}^{2}\right)},
$$

with an implied momentum conservation delta function as in Eq. (2.2). This result is in full agreement with the on-shell result in [7] (for $\alpha^{\prime} k_{1}^{2}=\alpha^{\prime} k_{2}^{2}=1$ and $\alpha^{\prime} k_{3}^{2}=0$ ), as well as the off-shell results summarized in [2]. To arrive at this result we needed to perform the computation of the following numerical coefficient:

$$
\begin{aligned}
b_{1} & =\sum_{e, e^{\prime}>0} T_{e 1} \sqrt{e}\left(\frac{2}{3+t \bar{t}}\right)_{e e^{\prime}} \frac{1}{\sqrt{e^{\prime}}} w_{e^{\prime}} \\
& =\left(\left(\frac{2}{3+\bar{t} t}\right) \kappa_{o}^{-1} v\right)_{1}=\frac{2 \sqrt{2}}{3 \sqrt{3}} .
\end{aligned}
$$

This computation, and those for similar quantities will be given later in this paper in Eq. (4.21).

We see that MSFT is in full agreement with previous computations in string field theory for off-shell 3-point functions. Previous methods used conformal maps [2] and conformal field theory, while MSFT uses the Moyal product, and produces the same results with considerably simpler methods. One can go on computing very simply off-shell 3-point couplings for any other perturbative or nonperturbative fields by similar MSFT techniques.

\section{OFF-SHELL 4-TACHYON SCATTERING}

One of the new results in our paper is a proof that the MSFT 4-tachyon amplitude produced by the Moyal product does give directly the Veneziano amplitude, without using conformal mapping techniques. A second new result is a comprehensive understanding of the off-shell factor by going considerably further in the parametric expansion, obtaining a plot of the function in the full range, and determining its differentiability at a turning point.

In MSFT we have been developing analytic methods of computation in string field theory by using directly the Moyal star product. Both the oscillator formalism and MSFT provide an expression for any off-shell amplitude, including loops. Although the starting point and intermediate steps are quite different, it has been argued that generally we expect agreement in the result $[13,15]$.

The computation of the 4-tachyon off-shell amplitude in MSFT was performed in $[13,15]$. There are 8 diagrams that correspond to various permutations of the four external legs. The $s$-channel diagram ${ }_{1}^{2}>-<_{4}^{3}$ is denoted as ${ }_{12} A_{34}$. The mathematical expression for this amplitude was obtained in $[13,15]$. The $t$-channel diagram is given by the cyclic permutation of the external legs ${ }_{41} A_{23}$, and its mathematical expression amounts to exchanging $s, t$ in the previous result. The remaining permutations that do not change the $s, t$ channel properties are denoted as ${ }_{21} A_{43},{ }_{43} A_{21},{ }_{34} A_{12}$ and ${ }_{14} A_{32}$, ${ }_{23} A_{41},{ }_{32} A_{14}$ respectively. After a brief computation (as in [13]) it can be shown that these give the same amplitudes as the initial $s, t$ diagrams. Therefore the sum of the diagrams for the $s, t$ channels produces a factor of 4

$$
\begin{aligned}
& A(s, t)=4\left(\begin{array}{ll}
{ }^{2} \vee^{3} \\
2>-<_{4}^{3}+ & \mid \\
& { }_{1} \wedge_{4}
\end{array}\right) \\
& =4 \int_{0}^{\infty} d \tau e^{\tau}\left({ }_{12} A_{34}(\tau)+{ }_{41} A_{23}(\tau)\right)
\end{aligned}
$$




$$
\begin{aligned}
= & \int_{0}^{\infty} d \tau e^{-\tau-2 \alpha(\tau)+4 \gamma(\tau)} a_{4}(\tau) \\
& \times \exp \left((\gamma(\tau)+2 \beta(\tau)) \sum_{i=1}^{4}\left(\alpha^{\prime} k_{i}^{2}-1\right)\right) \\
& \times\left[e^{\left(\alpha^{\prime} s+2\right)(\tau+\alpha(\tau)+2 \beta(\tau))}\right. \\
& \left.\times e^{\left(\alpha^{\prime} t+2\right) 2 \beta(\tau)}+(s \leftrightarrow t)\right]
\end{aligned}
$$

where $s, t$ are the Mandelstam variables ${ }^{3} s=-\left(k_{1}+k_{2}\right)^{2}$ and $t=-\left(k_{1}+k_{4}\right)^{2}$. The functions $\alpha(\tau), \beta(\tau), \gamma(\tau), a_{4}(\tau)$ initially given in [13] were further simplified in [15]. Here, as a first step, we simplify these functions further with a few algebraic steps and put them into the following form which will be convenient in our analysis

$$
\begin{aligned}
& \alpha(\tau)=\omega-\bar{b} e^{-\tau \kappa}\left(1-M e^{-\tau \kappa}\right)^{-1} b \\
& \beta(\tau)=\bar{b} P^{(o)} e^{-\tau \kappa}\left(1-M e^{-\tau \kappa} M e^{-\tau \kappa}\right)^{-1} P^{(o)} b \\
& \gamma(\tau)=-\frac{1}{2} \omega-\bar{b} P^{(o)} e^{-\tau \kappa}\left(1-M e^{-\tau \kappa}\right)^{-1} P^{(o)} b \\
& a_{4}(\tau)=4 \frac{1}{4} g^{2}\left(\frac{27}{16}\right)^{3} \frac{\operatorname{det}\left(1-\tilde{M} e^{-\tau \kappa} \tilde{M} e^{-\tau \kappa}\right)}{\left[\operatorname{det}\left(1-M e^{-\tau \kappa} M e^{-\tau \kappa}\right)\right]^{d / 2}}, d=26
\end{aligned}
$$

The overall factor of 4 in $a_{4}$ is the factor in Eq. (3.1). In string mode space labeled by even and odd positive integers $e=2,4,6, \ldots$ and $o=1,3,5, \ldots$, we have defined the matrices $\kappa, M, \tilde{M}, P^{(o)}$, vector $b$ and scalar $\omega$ as follows (a bar above a symbol means matrix transpose)

$$
\begin{aligned}
& \kappa=\left(\begin{array}{cc}
e & 0 \\
0 & o
\end{array}\right), M=\left(\begin{array}{cc}
\left(\frac{1-t \bar{t}}{3+t \bar{t}}\right)_{e e^{\prime}} & 0 \\
0 & \left(\frac{1-\bar{t} t}{3+\bar{t} t}\right)_{o o^{\prime}}
\end{array}\right), \\
& \tilde{M}=\left(\begin{array}{cc}
\left(\frac{1-t \bar{t}}{1+3 t \bar{t}}\right)_{e e^{\prime}} & 0 \\
0 & \left(\frac{1-\bar{t} t}{1+3 \bar{t} t}\right)_{o o^{\prime}}
\end{array}\right) \\
& b=\left(\begin{array}{l}
b_{e} \\
b_{o}
\end{array}\right), \quad P^{(o)}=\left(\begin{array}{ll}
0 & 0 \\
0 & 1
\end{array}\right), \quad \omega \equiv \bar{b}(1-M)^{-1} b .
\end{aligned}
$$

Here the diagonal matrix $\kappa$ with integer eigenvalues $\kappa_{n}$ $=n$, represents the spectrum of string oscillation frequencies

\footnotetext{
${ }^{3}$ The Mandelstam $t$ should not be confused with the matrix $t_{e o}$.
}

(eigenvalues of $\left.L_{0}\right), P^{(o)}$ is a projector onto the odd modes only. The other quantities are all constructed from the matrix $t_{e o}$ and vector $v_{o}$ which themselves are built from the frequencies $\kappa_{n}$ as given in [9-13] for any $N$. In the large $N$ limit they take the form in Eq. (4.3). The combination of $t_{e o}$ in the form of the matrix $M$ arises frequently in the interactions in the matter sector, while the matrix $\widetilde{M}$ occurs in the ghost sector. ${ }^{4}$ The even and odd vectors $b_{e}, b_{o}$ are given by

$$
b_{o}=\left(\frac{2}{3+\bar{t} t} \kappa_{o}^{-1 / 2} v\right)_{o}, \quad b_{e}=\left(t \frac{2}{3+t \bar{t}} \kappa_{o}^{-1 / 2} v\right)_{e} \text {, }
$$

where $\kappa_{o}$ is the odd part of the matrix $\kappa$. Finally $\omega$, which is the $\tau \rightarrow \infty$ limit of $\alpha(\tau)$ is simplified to the form

$$
\omega \equiv \bar{b}(1-M)^{-1} b=\bar{v} \kappa_{o}^{-1} \frac{2}{3+\bar{t} t} \kappa_{o}^{-1} v
$$

by using the definitions of $b, M$ given above.

From these quantities we compute the functions $\alpha(\tau), \beta(\tau), \gamma(\tau), a_{4}(\tau)$ which in turn determine the 4-tachyon scattering amplitude off-shell as well as on shell. We will show that the on-shell amplitude for $\alpha^{\prime} k_{i}^{2}=1$ reduces to the Veneziano amplitude given by the beta function

$$
A_{\text {shell }}(s, t)=g^{2} \frac{\Gamma\left(-\alpha^{\prime} s-1\right) \Gamma\left(-\alpha^{\prime} t-1\right)}{\Gamma\left(-\alpha^{\prime} s-\alpha^{\prime} t-2\right)} .
$$

On the other hand the off-shell expression above goes beyond conformal field theory which only gives information for on-shell strings.

The aim in the rest of this section is to show that the off-shell 4-tachyon amplitude in Eq. (3.1) can be rewritten in the following form and then compute the function $f(x)$

$$
\begin{aligned}
A(s, t)= & -g^{2} \int_{0}^{\infty} d \tau \frac{d x(\tau)}{d \tau}(f[x(\tau)])^{\sum_{i=1}^{4}\left(\alpha^{\prime} k_{i}^{2}-1\right)} \\
& \times\left[(x(\tau))^{-\alpha^{\prime} s-2}(1-x(\tau))^{-\alpha^{\prime} t-2}+(s \leftrightarrow t)\right] \\
= & g^{2} \int_{0}^{1} d x x^{-\alpha^{\prime} s-2}(1-x)^{-\alpha^{\prime} t-2}(f(x))^{\sum_{i=1}^{4}\left(\alpha^{\prime} k_{i}^{2}-1\right)}
\end{aligned}
$$

After the change of integration variables from $\tau$ to $x$ in the form of Eq. (3.12) we see that the off-shell amplitude is

\footnotetext{
${ }^{4}$ The matrices $M, \tilde{M}$ are simplified forms of $-\mathcal{M}^{(0)},-C X^{(0)}$ where $\mathcal{M}^{(0)}, X^{(0)}$ were identified in $[11,15]$ as some of the Neumann matrices for the 3-point vertex in the matter and ghost sectors respectively. Furthermore, it was shown that all Neumann matrices, for all $n$-point vertices, are explicit functions of the matrix $t_{e o}$ as obtained in $[11,15]$. Hence $t$ is the fundamental matrix that determines all interactions in string theory.
} 
consistent with the on-shell Veneziano amplitude [beta function in Eq. (3.10)] when $\alpha^{\prime} k_{i}^{2}=1$.

We will show that the change of variables from $\tau$ to $x$ is such that $x(0)=1 / 2$ and $x(\infty)=0$. Then we see that the $s$-channel amplitude ${ }_{12} A_{34}(\tau)={ }_{1}^{2}>--<_{4}^{3}$ contributes to the range $0 \leqslant x \leqslant 1 / 2$ while the $t$-channel amplitude ${ }_{41} A_{23}(\tau)$ contributes to the range $1 / 2 \leqslant x \leqslant 1$ after a change of variables $x \rightarrow(1-x)$. For the first form in Eq. (3.1) to agree with the second form in Eq. (3.11) it is required that $\alpha(\tau), \beta(\tau), \gamma(\tau), a_{4}(\tau)$ conspire to have remarkable relations among themselves so that they can be written as functions of the same $x(\tau)$. Thus we need to prove that the following relations are satisfied [which also define $x(\tau)$ in terms of $\alpha(\tau), \beta(\tau), \gamma(\tau), a_{4}(\tau)$ ]

$$
\begin{aligned}
e^{-\tau} e^{-\alpha(\tau)-2 \beta(\tau)} & =1-e^{-2 \beta(\tau)} \equiv x(\tau), \\
\frac{d x(\tau)}{d \tau} & =-\frac{a_{4}(\tau)}{g^{2}} e^{-\tau-2 \alpha(\tau)+4 \gamma(\tau)} .
\end{aligned}
$$

Note that $a_{4}(\tau)$ depends on $d=26$ while the other quantities are independent of the number of dimensions. Hence if the relation holds for $d=26$ it cannot hold for other dimensions. We will prove below that the relations in Eqs. (3.13), (3.14) are indeed true. This makes $d=26$ unique.

Once the relations are proven, then we learn that the offshell factor $f(x)$ is given by

$$
f(x(\tau))=e^{\gamma(\tau)+2 \beta(\tau)} .
$$

To write $f(x)$ in terms of only the parameter $x$ in the integral representation of the off-shell amplitude in Eq. (3.12), the relation between $x, \tau$ given in Eq. (3.13) needs to be inverted $\tau=\tau(x)$. We will perform the inversion and will construct the function $f(x)$ as a series expansion in powers of $x$. It turns out that a few terms in the expansion already give the necessary information to obtain a sufficiently accurate representation of the function $f(x)$, and hence of the full off-shell 4-tachyon amplitude.

Let us first prove that the relations in Eqs. (3.13) hold at the integration limits $\tau=0, \infty$. In the next section we show how to compute the functions $\alpha(\tau), \beta(\tau), \gamma(\tau), a_{4}(\tau)$ at $\tau$ $=0, \infty$. We find in particular

$$
\begin{aligned}
& \alpha(0)=0, \quad \beta(0)=\frac{1}{2} \ln 2, \quad \gamma(0)=-\ln 2, \\
& \alpha(\infty)=\ln \frac{27}{16}, \quad \beta(\infty)=0, \quad \gamma(\infty)=\frac{1}{2} \ln \frac{16}{27} \\
& a_{4}(\infty)=g^{2}\left(\frac{27}{16}\right)^{3}
\end{aligned}
$$

From this we see that indeed Eqs. (3.13) is satisfied at both limits $\tau=0, \infty$, and we also determine

$$
x(0)=\frac{1}{2}, \quad x(\infty)=0 .
$$

This shows that the $s$-channel amplitude ${ }_{12} A_{34}(\tau)$ is associated with the range $0 \leqslant x \leqslant 1 / 2$ and the $t$-channel amplitude ${ }_{41} A_{23}(\tau)$ contributes to the range $1 / 2 \leqslant x \leqslant 1$ after the change of variable $x \rightarrow(1-x)$.

Next we examine the relations for more general values of $\tau$. The form of the equations in Eqs. (3.2)-(3.5) was developed to examine an expansion in powers of $e^{-\tau}$. In the next section we show how to compute the coefficients for the large $\tau$ expansion of the functions $\alpha(\tau), \beta(\tau), \gamma(\tau), a_{4}(\tau)$. We find the following analytic result:

$$
\begin{aligned}
\alpha(\tau)= & -\ln \frac{16}{27}-\frac{8}{27} e^{-\tau}-\frac{2^{2} 19}{(27)^{2}} e^{-2 \tau}-\frac{2^{5} 7^{2}}{3(27)^{3}} e^{-3 \tau} \\
& -\frac{2 \times 13^{2} 43}{(27)^{4}} e^{-4 \tau}-\frac{2^{4} 167 \times 229}{5(27)^{5}} e^{-5 \tau}+O\left(e^{-6 \tau}\right),
\end{aligned}
$$

$$
\begin{aligned}
\beta(\tau)= & \frac{8}{27} e^{-\tau}+\frac{2^{5} 7^{2}}{3(27)^{3}} e^{-3 \tau}+\frac{2^{4} 167 \times 229}{5(27)^{5}} e^{-5 \tau} \\
& +O\left(e^{-7 \tau}\right),
\end{aligned}
$$

$$
\begin{aligned}
\gamma(\tau)= & \frac{1}{2} \ln \frac{16}{27}-\frac{8}{27} e^{-\tau}-\frac{40}{(27)^{2}} e^{-2 \tau}-\frac{2^{5} 7^{2}}{3(27)^{3}} e^{-3 \tau} \\
& -\frac{2^{3} 829}{(27)^{4}} e^{-4 \tau}-\frac{2^{4} 167 \times 229}{5(27)^{5}} e^{-5 \tau}+O\left(e^{-6 \tau}\right),
\end{aligned}
$$

$a_{4}(\tau)=g^{2}\left(\frac{27}{16}\right)^{3}\left(1+\frac{2^{2} 17}{3^{5}} e^{-2 \tau}+\frac{2 \times 1399}{(27)^{3}} e^{-4 \tau}+O\left(e^{-6 \tau}\right)\right)$.

To obtain the expansion to this order it is sufficient to compute the coefficients $b_{1}, b_{2}, b_{3}, b_{4}, b_{5}, M_{11}, M_{13}, M_{22}, \widetilde{M}_{11}$, $\widetilde{M}_{13}, \widetilde{M}_{22}$ defined above. These results were obtained analytically without much effort. Our analytic results above to order $O\left(e^{-6 \tau}\right)$ are already quite adequate to construct $f(x)$. It is possible to easily extend the expansion by inserting the results for the higher coefficients provided in the Appendix into Eqs. (3.2)-(3.5). In fact we have constructed an algebraic computer program that does this, and using it we have double checked our analytic results above and extended our computation to higher orders. We will report on some of the higher order results below.

The relations (3.13), (3.14) can now be verified directly by inserting the large $\tau$ expansions for $\alpha(\tau), \beta(\tau), \gamma(\tau), a_{4}(\tau)$ given above, and reexpanding in powers of $e^{-\tau}$ up to order $O\left(e^{-7 \tau}\right)$. From either the first or second term in Eq. (3.13) we obtain the same expression for $x(\tau)$, namely 


$$
\begin{aligned}
x(\tau) & =\left(\begin{array}{c}
\frac{16}{27} e^{-\tau}-\frac{128}{729} e^{-2 \tau}+\frac{64}{729} e^{-3 \tau}-\frac{19456}{531441} e^{-4 \tau} \\
+\frac{387296}{14348907} e^{-5 \tau}-\frac{1733120}{129140163} e^{-6 \tau}+O\left(e^{-7 \tau}\right)
\end{array}\right), \\
& =\left(\begin{array}{c}
59.259 e^{-\tau}-17.558 e^{-2 \tau}+8.7791 e^{-3 \tau}-3.6610 e^{-4 \tau} \\
+2.6991 e^{-5 \tau}-1.342 e^{-6 \tau}+O\left(e^{-7 \tau}\right)
\end{array}\right) \times 10^{-2} .
\end{aligned}
$$

Furthermore, the right hand side of Eq. (3.14) gives precisely the derivative $\partial_{\tau} x(\tau)$ of the expansion in Eq. (3.23)

$$
-\frac{a_{4}(\tau)}{g_{T}^{2}} e^{-\tau-2 \alpha(\tau)+4 \gamma(\tau)}=\left(\begin{array}{c}
-\frac{16}{27} e^{-\tau}+\frac{256}{729} e^{-2 \tau}-\frac{64}{243} e^{-3 \tau}+\frac{77824}{531441} e^{-4 \tau} \\
-\frac{1936480}{14348907} e^{-5 \tau}+\frac{3466240}{43046721} e^{-6 \tau}+O\left(e^{-7 \tau}\right)
\end{array}\right) .
$$

This proves that $\alpha(\tau), \beta(\tau)$ satisfy the relation in Eq. (3.13), and $\alpha(\tau), \gamma(\tau), a_{4}(\tau)$ satisfy the relation in Eq. (3.14), at least up to order $O\left(e^{-7 \tau}\right)$. Actually, as already mentioned, with a computer program we have shown that the relations hold to much higher orders. These results are convincing that $\alpha(\tau), \beta(\tau), \gamma(\tau), a_{4}(\tau)$ are all expressed in terms of the same function $x(\tau)$. We emphasize that the number of dimensions $d$ which appears in $a_{4}(\tau)$ in Eq. (3.5) must be $d$ $=26$ to satisfy the relations.

Note that at $\tau=0$ we expect $x(0)=1 / 2$ exactly as in Eq. (3.18), and indeed by replacing $e^{-\tau} \rightarrow 1$ the expansion (3.23) we obtain $x(0)=0.48176$ which implies that the expansion captures an accurate representation of the full function. Note from the trend in Eq. (3.24) that the next $O\left(e^{-7 \tau}\right)$ correction will bring the value much closer to the exact answer $x(0)$ $=1 / 2$. With the computer program we have shown that this value becomes $x(0)=0.49295$ by computing $x(\tau)$ to order $O\left(e^{-10 \tau}\right)$.

Finally we compute the off-shell factor, which is given by

$$
f(x(\tau))=e^{\gamma(\tau)+2 \beta(\tau)} \equiv \frac{C(1-x(\tau))}{2 \sqrt{1-x(\tau)}} .
$$

The expression on the right hand side defines the function $C$ which is given here for comparison to the old literature. Thus, by substituting $1-x(\tau)=\exp (-2 \beta(\tau))$ we can write $C(1-x(\tau))=2 e^{\gamma(\tau)+\beta(\tau)}$. First we obtain the expansion of $C(1-x(\tau))$ by inserting our computation of $\gamma(\tau)$ and $\beta(\tau)$. The result is

$$
\begin{aligned}
C(1-x(\tau))= & 2 e^{\gamma(\tau)+\beta(\tau)}=\left(\frac{4}{3}\right)^{3 / 2}\left(1-\frac{5}{32}\left(\frac{16}{27}\right)^{2} e^{-2 \tau}\right. \\
& \left.-\frac{1}{32}\left(\frac{16}{27}\right)^{2} e^{-4 \tau}+O\left(e^{-6 \tau}\right)\right) .
\end{aligned}
$$

Next, by using Eq. (3.23) we rewrite this result in terms of $x(\tau)$ so that the function $C(1-x(\tau))$ is given in terms of $x$. The result is

$$
\begin{aligned}
& C(1-x(\tau)) \\
& =\left(\frac{4}{3}\right)^{3 / 2}\left(\begin{array}{c}
1-\frac{5}{32}[x(\tau)]^{2}-\frac{5}{32}[x(\tau)]^{3} \\
-\frac{1249}{8192}[x(\tau)]^{4}-\frac{609}{4096}[x(\tau)]^{5}+O\left([x(\tau)]^{6}\right)
\end{array}\right) \\
& =\left(\begin{array}{c}
1.5396-0.24056 x^{2}-0.24056 x^{3} \\
-0.23474 x^{4}-0.22891 x^{5}+O\left(x^{6}\right)
\end{array}\right)
\end{aligned}
$$

These coefficients are determined by demanding that the expansion in powers of $e^{-\tau}$ matches the one of $2 e^{\gamma(\tau)+\beta(\tau)}$. In this form our result for $C$ is in agreement with what is found in the old literature [3] where $C$ was computed up to the second term $-5 / 32 x^{2}$ by using Mandelstam's conformal mapping techniques. We, of course, used the very different Moyal star technique and obtained the same result, but we also easily went further by obtaining the higher order terms, which is a new result given in this paper. As mentioned, with our technique it is very easy to compute to even higher orders [see Eq. (3.33)].

Since the range for $x$ is $0 \leqslant x \leqslant 1 / 2$ the expansion given above for $C$ has good convergence, so we expect that we have obtained an accurate representation of the full function $C$ in the relevant range. As a test let us compare the exact value of $C$ at $\tau=0$ (or at $x=1 / 2$ ) which we can compute exactly $C(1-x(0))=2 e^{\gamma(0)+\beta(0)}=\sqrt{2}=1.4142$ after using Eq. (3.16). By evaluating the expansion above at $x=1 / 2$, we obtain $C=1.4276$, which confirms that the expansion does capture the function almost fully in the entire range.

Let us now turn to the full off-shell factor $f(x)$. The exact expansion of this function in powers of $x$ up to $O\left(x^{6}\right)$ becomes 


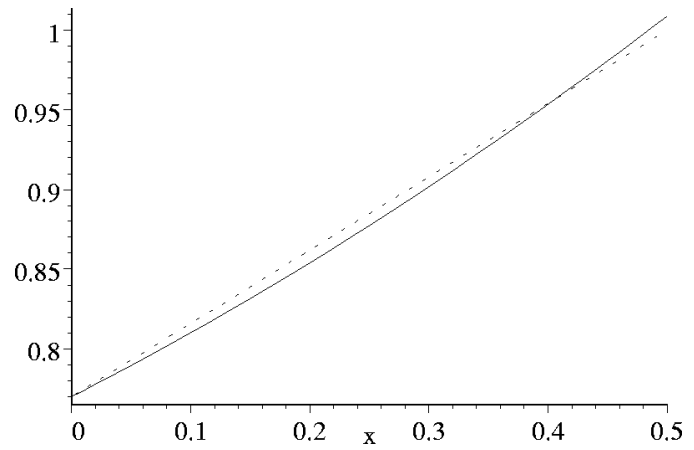

FIG. 1. Polynomial approximation to $f(x)$.

$$
\begin{aligned}
f(x) & =\frac{C(1-x)}{2(1-x)^{1 / 2}} \\
& =\frac{4}{3 \sqrt{3}}\left(\begin{array}{c}
1+\frac{1}{2} x+\frac{7}{32} x^{2}+\frac{5}{64} x^{3}-\frac{129}{8192} x^{4} \\
-\frac{1413}{16384} x^{5}+O\left(x^{6}\right)
\end{array}\right) \\
& =\frac{4}{3 \sqrt{3}}\left(\begin{array}{c}
1+0.5 x+0.21875 x^{2}+0.07813 x^{3} \\
-0.01575 x^{4}-0.08624 x^{5}+O\left(x^{6}\right)
\end{array}\right) .
\end{aligned}
$$

At the end of the range $x=1 / 2$ (i.e. $\tau=0$ ) the exact value of this function should be 1 , since

$$
f(1 / 2)=f(x(0))=e^{\gamma(0)+2 \beta(0)}=1,
$$

where we used Eq. (3.16). The series approximation in powers of $x$ given above to order $O\left(x^{5}\right)$ produces $f(1 / 2)$ $=1.0090$, which is better than $1 \%$ accuracy.

To get a better feel of the function $f(x)$ we plot the expansion in Eq. (3.29) for the appropriate range for $x$ which is $0 \leqslant x \leqslant 1 / 2$. This is shown by the solid line in Fig. 1 . The figure suggests that, for the relevant range, as a guide for the eye we can compare $f(x)$ roughly to a linear function $\widetilde{f}(x)$

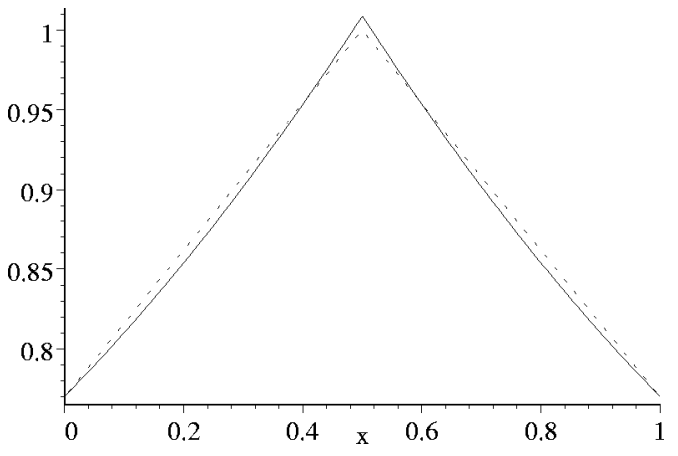

FIG. 2. Polynomial approximation to $f(x)$.

$$
\widetilde{f}(x)=\frac{4}{3 \sqrt{3}}+2\left(1-\frac{4}{3 \sqrt{3}}\right) x,
$$

where the slope is chosen to guarantee the exact values at both ends of the range, namely $4 / 3 \sqrt{3}$ at $x=0$ and 1 at $x$ $=1 / 2$. This case corresponds to the dotted line in the figure. The plots suggest that the exact curve $f(x)$ lies somewhere close to the solid line and the dotted line, and that in any case we have obtained a fairly good approximation in the entire range with the series expansion in Eq. (3.29).

By combining the $s$ and $t$ channel contributions we extend the range of integration to $0 \leqslant x \leqslant 1$ as explained above. Thus, in the range $1 / 2 \leqslant x \leqslant 1$ the function $f(x)$ is given by substituting $x$ by $(1-x)$ in the expression of $f(x)$ given above. Namely, the plot of $f(x)$ in the full range $0 \leqslant x \leqslant 1$ is given in Fig. 2.

The exact function is expected to lie somewhere close to the solid and the dotted lines. The shape of the curve begs the question of whether the exact $f(x)$ ever crosses the dotted line. To provide an answer we used our algebraic computer program in which we plugged in the contents of the Appendix into Eqs. (3.2)-(3.5) and obtained the following higher order expansion [we do not give the details of the expansion for $\left.\alpha(\tau), \beta(\tau), \gamma(\tau), a_{4}(\tau)\right]$

$$
f(x)=\frac{4}{3 \sqrt{3}}\left(\begin{array}{c}
1+\frac{1}{2} x+\frac{7}{32} x^{2}+\frac{5}{64} x^{3}-\frac{129}{8192} x^{4}-\frac{1413}{16384} x^{5}-\frac{40973}{262144} x^{6} \\
-\frac{124459}{524288} x^{7}-\frac{186841777}{536870912} x^{8}-\frac{547864633}{1073741824} x^{9}+O\left(x^{10}\right)
\end{array}\right)
$$

The plot of this more accurate expansion lies slightly below the solid line in the figure, and is extremely close to it in the regions that are not near to $x=1 / 2$. It does cross the dotted line, but it does it at mirror points closer to $x=1 / 2$ compared to Fig. 2, and finally reaches the value $f(1 / 2)=1.0039$ at $x=1 / 2$. So the new corrected peak is between the previous value of 1.0090 and the exact value $1.0090>1.0039>1$. This analysis is consistent with the possibility that the exact function $f(x)$ may cross the dotted line somewhere close to $x=1 / 2$ before settling into the value $f(1 / 2)=1$. This issue of crossing or not crossing is relevant for determining whether the exact function is differentiable at $x=1 / 2$. If it never 
crosses the dotted line then it must make a cusp and be nondifferentiable. Such a nondifferentiable function may be a peculiarity of Witten's theory in which a particular conformal gauge has been effectively fixed by distinguishing the midpoint. We remind the reader that off-shell amplitudes generally are not gauge invariant.

It seems therefore interesting to investigate the slope of $f(x)$ near $x=1 / 2$. A glimpse of the slope on the left side of the possible cusp is obtained by computing the derivative of the expansion above and evaluating it at $x=1 / 2$. We obtain $\left.\partial_{x} f(x)\right|_{x=1 / 2} \simeq 0.49849$ consistent with a cusp. However it is not clear how much we can trust this number, because if we examine the size of the coefficients in the expansion of the derivative

$$
\partial_{x} f(x)=\left(\begin{array}{c}
38.49+16.839(2 x)+4.5105(2 x)^{2}-0.60611(2 x)^{3}-2.0747(2 x)^{4} \\
-2.2560(2 x)^{5}-1.9987(2 x)^{6}-1.6744(2 x)^{7}-1.3809(2 x)^{8}+O\left(x^{9}\right)
\end{array}\right) \times 10^{-2}
$$

we see that convergence is not very fast, and the exact value may turn out to be quite different at $2 x=1$ since there are an infinite number of terms to be summed. Note that the tendency of the neglected terms is to be negative thereby reducing the value of the slope given above. To settle the question we must examine an expansion near $\tau=0$ rather than near $\tau=\infty$, but the reason we did not do this so far in this paper is because the expansion does not seem to exist due to divergent derivatives near $\tau=0$. However, precisely these divergences turn out to settle the issue as follows.

We can compute the derivative $\left(\partial_{x} f(x)\right)_{x=1 / 2}$ from $\left.\partial_{\tau} f(x(\tau))\right|_{\tau=0}$ as follows

$$
\begin{aligned}
\dot{x}(0)\left(\partial_{x} f(x)\right)_{x=1 / 2} & =\left.\partial_{\tau} f(x(\tau))\right|_{\tau=0} \\
& =(\dot{\gamma}(0)+2 \dot{\beta}(0)) e^{\gamma(0)+2 \beta(0),}
\end{aligned}
$$

where we have used the chain rule on the left hand side and Eq. (3.26) on the right hand side. From Eq. (3.13) we have $\dot{x}(0)=2 \dot{\beta}(0) e^{-2 \beta(0)}$. Inserting the exact values for $\gamma(0), \beta(0)$ given in Eq. (3.16) these equations become

$$
\dot{x}(0)=\dot{\beta}(0), \quad \dot{x}(0)\left(\partial_{x} f(x)\right)_{x=1 / 2}=\dot{\gamma}(0)+2 \dot{\beta}(0) .
$$

By differentiating Eqs. (3.3), (3.4) we obtain expressions for $\dot{\gamma}(0), \dot{\beta}(0)$

$$
\begin{aligned}
& \dot{\beta}(0)=-\bar{b}_{o}\left(1-M_{o}^{2}\right)^{-1}\left(\kappa_{o}+M_{o} \kappa_{o} M_{o}\right)\left(1-M_{o}^{2}\right)^{-1} b_{o}, \\
& \dot{\gamma}(0)=\bar{b}_{o}\left(1-M_{o}\right)^{-1} \kappa_{o}\left(1-M_{o}\right)^{-1} b_{o},
\end{aligned}
$$

where $M_{o}=(1-\bar{t} t) /(3+\bar{t} t)$ is the odd part of the matrix $M$. After straightforward algebra, we find

$$
\dot{\gamma}(0)=\bar{v} \kappa_{o}^{-1 / 2} \frac{1}{(1+\bar{t} t)} \kappa_{o} \frac{1}{(1+\bar{t} t)} \kappa_{o}^{-1 / 2} v,
$$

and $\dot{x}(0), \dot{\beta}(0)$ related to the same expression by

$$
\dot{x}(0)=\dot{\beta}(0)=-\frac{1}{2} \dot{\gamma}(0)-\frac{1}{2} .
$$

We used $\bar{v} v=1$ which multiplied the last constant term $-1 / 2$. In passing we mention that we have also computed $\dot{\alpha}(0)$ and found $\dot{\alpha}(0)=2 \dot{\gamma}(0)$ after a little algebra. Through Eqs. (4.16), (4.17) and Eqs. (5.36)-(5.40) in the next section we show that the expression above for $\dot{\gamma}(0)$ is divergent at large $N$. So $\dot{\gamma}(0)+2 \dot{\beta}(0)=-1$ is finite, but $\dot{x}(0)$ diverges. Therefore from Eq. (3.36) we find that the slope of $f(x)$ at $x=1 / 2$ is exactly zero

$$
\left(\partial_{x} f(x)\right)_{x=1 / 2}=0 .
$$

So, after all the off-shell function $f(x)$ is continuous and differentiable at $x=1 / 2$. With this result on the slope, the expansion in Eq. (3.33), and the plot in Fig. 2, we have basically understood the function $f(x)$.

This discussion provides the most comprehensive result for the off-shell scattering amplitude of four tachyons produced so far in string field theory.

\section{COMPUTATIONAL TECHNIQUES}

In this paper we need to evaluate the quantities $\omega, b_{o}, b_{e}, c_{e}, c_{o}, M_{e e^{\prime}}, M_{o o^{\prime}}, \widetilde{M}_{e e^{\prime}}, \widetilde{M}_{o o^{\prime}}$, and various determinants, as defined in the previous sections. These are examples of more general computations that come up in MSFT which are generically of the type

$$
\begin{aligned}
& (F(\bar{t} t))_{o o^{\prime}},(F(t \bar{t}))_{e e^{\prime}},(t F(\bar{t} t))_{e o}, \\
& \left(F(\bar{t} t) \kappa^{-1 / 2} v\right)_{o},\left(t F(\bar{t} t) \kappa^{-1 / 2} v\right)_{e} \\
& \operatorname{det}(F(\bar{t} t)), \quad \bar{v} \kappa_{o}^{-1 / 2} F(\bar{t} t) \kappa_{o}^{-1 / 2} v, \\
& \bar{v} \kappa_{o}^{-1 / 2} F(\bar{t} t) \kappa_{o} F(\bar{t} t) \kappa_{o}^{-1 / 2} v, \text { etc. }
\end{aligned}
$$

where $F(z)$ can be any function of the matrix $\bar{t} t$ or $t \bar{t}$. In the present paper the functions $F$ of interest are $M$ $=(1-\bar{t} t) /(3+\bar{t} t)$ and $\widetilde{M}=(1-\bar{t} t) /(1+3 \bar{t} t)$ and other 
simple ones constructed from them, such as $4 /(3+\bar{t} t)=1$ $-M,\left(1-M^{2}\right)$, etc. As seen in the full $\tau$-dependent $\alpha(\tau), \beta(\tau)$ etc. there are more involved combinations of $F(\bar{t} t)$ and the matrix $e^{-\tau \kappa}$ which can appear in explicit calculations. The techniques discussed below do not directly apply to compute such quantities fully, but do apply to compute the expansion coefficients analytically and exactly when these quantities are expanded in powers of $e^{-\tau}$. This is how we made progress in this paper.

In our MSFT approach such quantities are initially defined in terms of the regulated vector $v_{o}$ and the regulated matrix $t_{e o}$. By inserting the values of $v_{o}, t_{e o}$ for any number of modes $2 N$, all of these quantities take on explicit numerical values. Generally it is seen that a few modes already give an answer pretty close to the $N \rightarrow \infty$ limit, therefore for a quick estimate of nondivergent quantities it is generally sufficient to use finite matrices for just a few modes $N$. With this approach we can evaluate any quantity approximately fairly easily.

The large $N$ limit is sometimes subtle because of anomalies. Generally anomalies arise in the form of a zero multiplied by an infinity that comes from an infinite sum over matrix indices (the modes) producing $0 \times \infty$, so that there is a subtle finite contribution. If one is not careful the zero is first evaluated and the infinite sum that is later performed still gives zero, thus missing the finite contribution. To avoid anomaly subtleties in the $N \rightarrow \infty$ limit we use the regulated version of $v_{o}, t_{e o}$ in all computations (see $[10,15]$ ), and take the $N \rightarrow \infty$ only at the end of the computation. The regulated matrices $v_{o}, t_{e o}$ satisfy certain algebraic relations, also shared by the unregulated matrices, that make it possible to perform certain computations analytically. It has been demonstrated that this is a correct regulator consistent with other computational techniques in string theory.

In certain computations, or parts of it, there are no anomalies, and it becomes possible to work with the infinite unregulated matrices $v_{e}, t_{e o}$ directly at $N=\infty$. Then it is possible to make a transformation to the basis that diagonalizes the infinite unregulated matrix $t_{e o}$ and do the computations in the $N=\infty$ diagonal basis. At $N=\infty$ the eigenvalues of $t_{e o}$ are continuous and are parameterized by a continuous variable $\kappa$, and furthermore the transformation is known explicitly [16]. As we will discuss in detail in the next section, at $N$ $=\infty$ the discrete and continuous bases are just different bases of $\operatorname{SL}(2, R)$ in the $j(j+1)=0$ unitary representation, in which $t_{e o}$ can be understood as the matrix elements of the operator $t=\tanh \left((\pi / 4)\left(L_{1}+L_{-1}\right)\right)$ in the discrete basis that diagonalizes $L_{0}$. The continuous basis is the basis in which this operator is diagonal.

A priori it is hard to know when to expect an anomaly if one works directly at $N=\infty$. Therefore the regulator provided in MSFT is indispensable to insure that such subtleties will not spoil a computation. So one must be wary when using the continuous basis directly at $N=\infty$. We emphasize that the discrete Moyal basis with a regulator is the safe way to proceed in general, but one can make a transformation to the continuous basis for specific computations to evaluate infinite sums in parts of computations. This is the sense in which we use the continuous basis in the next subsection, and indeed we will find good use for it in some of the following computations.

\section{A. Computations via transformation to continuous basis}

In the following computations we are directly at $N=\infty$ and work with the unregulated fundamental matrices $T, R, v, w$ in MSFT that encode string joining (for a particular regularization see footnote 6 )

$$
w_{e}=\sqrt{2}(i)^{-e+2}, \quad v_{o}=\frac{2 \sqrt{2}}{\pi} \frac{(i)^{o-1}}{o}, \quad t_{e o}=\sqrt{e} T_{e o} \frac{1}{\sqrt{o}},
$$

$T_{e o}=\frac{4 o(i)^{o-e+1}}{\pi\left(e^{2}-o^{2}\right)}, \quad R_{o e}=\frac{4 e^{2}(i)^{o-e+1}}{\pi o\left(e^{2}-o^{2}\right)}$.

As explained in $[11,12]$, the matrix $t$ is diagonalized $t_{e o}$ $=\left(V_{e} \tau \bar{V}_{o}\right)_{e o}$ by the orthogonal matrices $V_{o}, V_{e}$ that act on the odd or even sides (a bar means transpose). The eigenspace is labeled by $\kappa$ with eigenvalues ${ }^{5} \tau_{\kappa}$. The label $\kappa$ is continuous (can happen only for infinite matrices); it is in the range $0 \leqslant \kappa \leqslant \infty$, and the eigenvalues are given by $[21,16]$ $\tau_{\kappa}=\tanh (\pi \kappa / 4)$. The orthogonal matrices have matrix elements which are functions of $\kappa$ and the index $o, e$, namely $\left(V_{o}\right)_{o \kappa} \equiv V_{o}(\kappa)$, and $\left(V_{e}\right)_{e \kappa}=V_{e}(\kappa)$. Thus the matrix equation $t_{e o}=\left(V_{e}\right)_{e \kappa} \tau_{\kappa}\left(\bar{V}_{o}\right)_{\kappa o}$ is written as

$$
t_{e o}=\int_{0}^{\infty} d \kappa V_{e}(\kappa) \tanh (\pi \kappa / 4) V_{o}(\kappa)
$$

The functions $V_{e}(\kappa), V_{o}(\kappa)$ satisfy orthogonality relations that correspond to the orthogonality conditions on the matrices $V_{e}, V_{o}$. These functions are given explicitly by the generating functions in Eq. (5.3). In the next section we will clarify the role of these functions as the overlaps $\langle e \mid \kappa\rangle$ $=V_{e}(\kappa) / \sqrt{2}$ and $\langle o \mid \kappa\rangle=V_{o}(\kappa) / \sqrt{2}$ between the states of two different bases of the $j=0$ representation of $\operatorname{SL}(2, R)$ generated by the Virasoro generators $L_{0}, L_{ \pm 1}$. The first basis is the familiar one $|n\rangle$ labeled by the even or odd eigenvalues $n=(e, o)$ of $L_{0}$. The second basis $|\kappa\rangle$ diagonalizes $\left(L_{+1}\right.$ $\left.+L_{-1}\right)|\kappa\rangle=\kappa|\kappa\rangle$.

By evaluating the generating functions in Eq. (5.3) at $\kappa$ $=0$ and expanding in powers of $z$, one can see that $V_{e}(0)$ $=0$, while $V_{o}(0)$ is finite and related to $v_{o}$ as $V_{o}(0)$ $=\frac{1}{2} \sqrt{\pi o} v_{o}$. Thus $v_{o}$ is directly related to the state $|\kappa=0\rangle$. We have evaluated the nontrivial infinite sum

\footnotetext{
${ }^{5}$ This continuous parameter $\kappa$ should not be confused with the diagonal matrix $\kappa$ of Eq. (3.6). As much as possible we are trying to keep the notations that were introduced in different papers by independent authors. We hope the reader will discern them in the proper contexts.
} 
$\Sigma_{o}\left[V_{o}(\kappa) / \sqrt{o}\right] v_{o}$ in the next section. We give it below along with a few of the $V_{e}(\kappa), V_{o}(\kappa)$ that are needed in our computations

$$
\begin{gathered}
V_{1}(\kappa)=\sqrt{\frac{\kappa}{\sinh \frac{\pi \kappa}{2}}}, V_{2}(\kappa)=\frac{\kappa^{2}}{\sqrt{2 \kappa \sinh \frac{\pi \kappa}{2}}}, \\
V_{3}(\kappa)=\frac{-\kappa+\frac{1}{2} \kappa^{3}}{\sqrt{3 \kappa \sinh \frac{\pi \kappa}{2}}}, \\
V_{4}(\kappa)=\frac{\frac{1}{6} \kappa^{2}\left(\kappa^{2}-8\right)}{\sqrt{4 \kappa \sinh \frac{1}{2} \pi \kappa}}, \quad V_{5}(\kappa)=\frac{\kappa\left(1-\frac{5}{6} \kappa^{2}+\frac{1}{24} \kappa^{4}\right)}{\sqrt{5 \kappa \sinh \frac{1}{2} \pi \kappa}}, \\
\sum_{o} \frac{V_{o}(\kappa)}{\sqrt{o} v_{o}}=\sqrt{\frac{\tanh \frac{\pi \kappa}{4}}{\kappa \cosh ^{2} \frac{\pi \kappa}{4}} .}
\end{gathered}
$$

With this preparation we are ready to compute the desired generic quantities. Applying the diagonalization of $t_{e o}$ we have just described we can write $t=V_{e} \tau \bar{V}_{o}, \bar{t} t=V_{o} \tau^{2} \bar{V}_{o}$ and $t \bar{t}=V_{e} \tau^{2} \bar{V}_{e}$ and express the desired quantities as integrals

$$
\begin{aligned}
(F(\bar{t} t))_{o o^{\prime}}= & \int_{0}^{\infty} d \kappa V_{o}(\kappa) F\left(\tanh ^{2}\left(\frac{\pi \kappa}{4}\right)\right) V_{o^{\prime}}(\kappa) \\
(F(t \bar{t}))_{e e^{\prime}}= & \int_{0}^{\infty} d \kappa V_{e}(\kappa) F\left(\tanh ^{2}\left(\frac{\pi \kappa}{4}\right)\right) V_{e^{\prime}}(\kappa) \\
(t F(\bar{t} t))_{e o}= & \int_{0}^{\infty} d \kappa V_{e}(\kappa) \tanh \left(\frac{\pi \kappa}{4}\right) F\left(\tanh ^{2}\left(\frac{\pi \kappa}{4}\right)\right) \\
& \times V_{o}(\kappa)
\end{aligned}
$$

$$
\begin{aligned}
\left(F(\bar{t} t) \kappa^{-1 / 2} v\right)_{o}= & \int_{0}^{\infty} d \kappa V_{o}(\kappa) F\left(\tanh ^{2}\left(\frac{\pi \kappa}{4}\right)\right) \\
& \times \sqrt{\frac{\tanh \frac{\pi \kappa}{4}}{\kappa \cosh ^{2} \frac{\pi \kappa}{4}}}
\end{aligned}
$$

$$
\left(t F(\bar{t} t) \kappa^{-1 / 2} v\right)_{e}=\int_{0}^{\infty} d \kappa V_{e}(\kappa) \tanh \left(\frac{\pi \kappa}{4}\right)
$$$$
\times F\left(\tanh ^{2}\left(\frac{\pi \kappa}{4}\right)\right) \sqrt{\frac{\tanh \frac{\pi \kappa}{4}}{\kappa \cosh ^{2} \frac{\pi \kappa}{4}}}
$$

$$
\begin{aligned}
\bar{v} \kappa_{o}^{-1 / 2} F(\bar{t} t) \kappa_{o}^{-1 / 2} v= & \int_{0}^{\infty} d \kappa \sqrt{\frac{\tanh \frac{\pi \kappa}{4}}{\kappa \cosh ^{2} \frac{\pi \kappa}{4}}} \\
& \times F\left(\tanh ^{2}\left(\frac{\pi \kappa}{4}\right)\right) \sqrt{\frac{\tanh \frac{\pi \kappa}{4}}{\kappa \cosh ^{2} \frac{\pi \kappa}{4}}} \\
\operatorname{det} F(\bar{t} t)= & \exp \int_{0}^{\infty} d \kappa \rho(\kappa) \ln \left[F\left(\tanh ^{2}\left(\frac{\pi \kappa}{4}\right)\right)\right]
\end{aligned}
$$

In the infinite $N$ limit we have $\operatorname{det}(F(t \bar{t}))=\operatorname{det}(F(\bar{t} t))$. The quantity

$$
\rho(\kappa)=\left(\frac{1}{2 \pi} \ln \left(2 N e^{\Delta}\right)-\frac{1}{4 \pi}\left[\psi\left(\frac{i \kappa}{2}\right)+\psi\left(-\frac{i \kappa}{2}\right)\right]\right)
$$

which appears in the evaluation of determinants will be derived in the next section in Eq. (5.58). Here $\psi(z)$ $=\partial_{z} \ln \Gamma(z)$ is the logarithmic derivative of the gamma function, and $\Delta$ depends on the regulator. It could be chosen as zero if we compare to a particular regulator in the discrete basis of MSFT. The same result has been obtained in a very different type of calculation in $[25,26]$. The term that contains the number of modes $2 \mathrm{~N}$ is the leading term independent of $\kappa$. In physical computations, as $N$ goes to infinity this factor cancels magically among matter and ghost determinants as long as $d=26$, and the bare coupling $g_{0}$ is rewritten in terms of the on-shell tachyon coupling $g$. Hence the nonleading part in terms of the function $\psi(z)$ is the crucial part that contributes in physical processes.

A more complicate type of quantity that we needed in Eq. (3.39) takes the form of a double integral 


$$
\bar{v} \kappa_{o}^{-1 / 2} F(\bar{t} t) \kappa_{o} F(\bar{t} t) \kappa_{o}^{-1 / 2} v=\int_{0}^{\infty} d \kappa \int_{0}^{\infty} d \kappa^{\prime}
$$

where

$$
\begin{aligned}
\left(\kappa_{o}\right)_{\kappa \kappa^{\prime}} & =\sum_{o} V_{o}(\kappa) o V_{o}\left(\kappa^{\prime}\right) \\
& =\frac{1}{2}\left\langle\kappa\left|L_{0}\right| \kappa^{\prime}\right\rangle+\frac{1}{2}\left\langle\kappa\left|L_{0}\right|-\kappa^{\prime}\right\rangle .
\end{aligned}
$$

The form of $\left(\kappa_{o}\right)_{\kappa \kappa^{\prime}}$ in terms of matrix elements of $L_{0}$ in the $\kappa$ basis is shown in the next section. We apply this formula to compute $\dot{\gamma}(0)$ in Eq. (3.39). The integrations in Eq. (4.16) are well behaved for $F(\bar{t} t)=(1+\bar{t} t)^{-1}$. But the infinite sum $\left(\kappa_{o}\right)_{\kappa \kappa^{\prime}}$ is shown to diverge in Eqs. (5.36)-(5.40). It is evaluated there more carefully by inserting a regulator in the form $\left(e^{-\tau \kappa_{o}} \kappa_{o}\right)_{\kappa \kappa^{\prime}}$. It is finite at finite $\tau$, but when $\tau$ is small it behaves like $1 / \tau$ times a quickly oscillating factor. The quick oscillations are not sufficient to overcome the divergence of the $1 / \tau$ factor. Therefore, this leads to the divergent result for $\dot{\gamma}(0)$ in Eq. (3.39) which we used in the previous section.

These integrals seem difficult, but can be easily performed with an algebraic computer program, such as Maple or Mathematica. For example we obtain

$$
\begin{aligned}
& \int_{0}^{\infty} d \kappa V_{1}(\kappa) \frac{2}{3+\tanh ^{2} \frac{\pi \kappa}{4}} \sqrt{\frac{\tanh \frac{\pi \kappa}{4}}{\kappa \cosh ^{2} \frac{\pi \kappa}{4}}}=0.54433 \\
&=\frac{2 \sqrt{2}}{3 \sqrt{3}} \\
& \int_{0}^{\infty} d \kappa V_{2}(\kappa) \frac{2 \tanh \frac{\pi \kappa}{4}}{3+\tanh ^{2} \frac{\pi \kappa}{4}} \sqrt{\frac{\tanh \frac{\pi \kappa}{4}}{\kappa \cosh ^{2} \frac{\pi \kappa}{4}}}=0.22222 \\
&=\frac{2}{9}
\end{aligned}
$$

$$
\begin{gathered}
\sqrt{\frac{\tanh \frac{\pi \kappa}{4}}{\kappa \cosh ^{2} \frac{\pi \kappa}{4}} F\left(\tanh ^{2}\left(\frac{\pi \kappa}{4}\right)\right)\left(\kappa_{o}\right)_{\kappa \kappa^{\prime}}} \\
F\left(\tanh ^{2}\left(\frac{\pi \kappa^{\prime}}{4}\right)\right) \sqrt{\frac{\tanh \frac{\pi \kappa^{\prime}}{4}}{\kappa \cosh ^{2} \frac{\pi \kappa^{\prime}}{4}}}
\end{gathered}
$$

$$
\begin{aligned}
& \int_{0}^{\infty} d \kappa \sqrt{\frac{\tanh \frac{\pi \kappa}{4}}{\kappa \cosh ^{2} \frac{\pi \kappa}{4}}} \frac{2}{3+\tanh ^{2} \frac{\pi \kappa}{4}} \sqrt{\frac{\tanh \frac{\pi \kappa}{4}}{\kappa \cosh ^{2} \frac{\pi \kappa}{4}}} \\
& =0.52325=\ln \frac{27}{16} .
\end{aligned}
$$

We now turn to the more specialized cases of relevance for our computations in this paper. We would like to evaluate the scalar $\omega=\bar{v} \kappa_{o}^{-1 / 2}[2 /(3+\bar{t} t)] \kappa_{o}^{-1 / 2} v$, the vectors $b_{o}$ $=\left([2 /(3+\bar{t} t)] \kappa_{o}^{-1 / 2} v\right)_{o}$ and $b_{e}=\left(t[2 /(3+\bar{t} t)] \kappa_{o}^{-1 / 2} v\right)_{e}$ and matrix elements $M_{o o^{\prime}}=((1-\bar{t} t) /(3+\bar{t} t))_{o o^{\prime}}, M_{e e^{\prime}}=((1$ $-t \bar{t}) /(3+t \bar{t}))_{e e^{\prime}}, \quad \tilde{M}_{o o^{\prime}}=((\bar{t} t-1) /(1+3 \bar{t} t))_{o o^{\prime}}, \quad \tilde{M}_{e e^{\prime}}$ $=((t \bar{t}-1) /(1+3 t \bar{t}))_{e e^{\prime}}$. By applying the above integral formulas we obtained easily the following values which are the only ones we actually needed in this paper to perform the expansions of $\alpha(\tau), \beta(\tau), \gamma(\tau), a_{4}(\tau)$ in powers of $e^{-\tau}$ up to order $O\left(e^{-6 \tau}\right)$

$$
\begin{aligned}
& b_{1}=\frac{2 \sqrt{2}}{3 \sqrt{3}}, \quad b_{2}=\frac{2}{9}, \quad b_{3}=-\frac{22 \sqrt{2}}{3^{5}}, \quad b_{4}=-\frac{19 \sqrt{2}}{3^{5}}, \\
& b_{5}=\frac{2^{3 / 2} 67}{(27)^{2} \sqrt{15}}
\end{aligned}
$$

$$
M_{11}=\frac{5}{27}, \quad M_{22}=\frac{13}{3^{5}}, \quad M_{13}=-\frac{2^{5} \sqrt{3}}{(27)^{2}}, \quad \omega=\ln \frac{27}{16},
$$

$\widetilde{M}_{11}=\frac{11}{27}, \quad \widetilde{M}_{22}=\frac{19}{9(27)}, \quad \widetilde{M}_{13}=-\frac{2^{4} 5 \sqrt{3}}{(27)^{2}}$.

We also show how to compute some determinants by using Eq. (4.14). We especially make a point to separate the contribution associated with the regulator $N$ which will be sent to infinity later. The steps of computation are shown for the following explicit example 


$$
\begin{aligned}
\operatorname{det}((t \bar{t})) & =e^{1 /(2 \pi) \ln \left(2 N e^{\Delta}\right) \int_{0}^{\infty} \ln \left(\tanh ^{2}(\pi \kappa / 4)\right) d \kappa} \times e^{\int_{0}^{\infty}(-(1 / 4 \pi)[\psi(i \kappa / 2)+\psi(-i \kappa / 2)]) \ln \left(\tanh ^{2}(\pi \kappa / 4)\right) d \kappa} \\
& =\left(2 N e^{\Delta}\right)^{-1 / 2}(0.79788)=\left(2 N e^{\Delta}\right)^{-1 / 2} \sqrt{\frac{2}{\pi}}=\left(\pi N e^{\Delta}\right)^{-1 / 2}
\end{aligned}
$$

As seen, the finite factor that comes from the nonleading term involving $\psi$ is nontrivial. We will show later in Eq. (5.62) how both the leading and nonleading terms agree with the regulated discrete basis. In a similar way we obtain

$$
\begin{aligned}
\operatorname{det}\left(\frac{3+(t \bar{t})}{4}\right) & =\left(2 N e^{\Delta}\right)^{-1 / 18}(0.98052) \\
\operatorname{det}\left(\frac{1+3(t \bar{t})}{4}\right) & =\left(2 N e^{\Delta}\right)^{-2 / 9}(0.91746) \\
\operatorname{det}\left(1-\left(\frac{1-t \bar{t}}{3+t \bar{t}}\right)^{2}\right) & =\left(2 N e^{\Delta}\right)^{-1 / 72}(0.99340) \\
\operatorname{det}\left(1-\left(\frac{1-\bar{t} t}{1+3 \bar{t} t}\right)^{2}\right) & =\left(2 N e^{\Delta}\right)^{-13 / 72}(0.90534)
\end{aligned}
$$

Note the magical number 26 that popped up as $13=26 / 2$ in the last ghost determinant. Inserting these determinants in the expression for $a_{4}$ in Eq. (3.5) we see that the $N$ dependence drops out at $\tau=0$, only for $d=26$,

$$
\begin{aligned}
a_{4}(0) & =g^{2}\left(\frac{27}{16}\right)^{3} \frac{\left(\left(2 N e^{\Delta}\right)^{-13 / 72}(0.90534)\right)^{2}}{\left[\left(2 N e^{\Delta}\right)^{-1 / 72}(0.99340)\right]^{26}} \\
& =g^{2}\left(\frac{27}{16}\right)^{3}(0.97363) .
\end{aligned}
$$

So the result is independent of the cutoff $N, \Delta$. This is an example of the magical role of $d=26$. Note that $a_{4}$, as given initially in $[13,15]$ has factors of additional determinants that are actually divergent, but those get absorbed into the definition of $g$ and produce the overall finite factor $g^{2}(27 / 16)^{3} / 4$ (multiplied by another factor of 4 because of 4 diagrams).

In the case of the bare coupling in Eq. (2.3), inserting the results above we get

$$
\begin{aligned}
g_{0}= & \frac{1}{2} g\left(\frac{27}{16}\right)^{3 / 2}\left(\left(2 N e^{\Delta}\right)^{-1 / 2}(0.79788)\right)^{-(d-6) / 4} \\
& \times\left((2 N)^{-1 / 18}(0.98052)\right)^{d}\left((2 N)^{-2 / 9}(0.91746)\right)^{-2} \\
= & g c\left(2 N e^{\Delta}\right)^{3 / 2} \\
& \text { for } d=26, \text { with } c=\frac{1}{2}\left(\frac{27}{16}\right)^{3 / 2}(2.2030)
\end{aligned}
$$

We see that the bare coupling diverges as $(N)^{3 / 2}$.
In the Appendix we have included many more values for the higher modes of the vectors $b$ and matrices $M, \tilde{M}$. Although these were not needed for our analytic calculation, they were used to obtain the order ten result for $f(x)$. Also the general formulas we give there are useful to compute to arbitrary powers of $e^{-\tau}$ if one needs to refine our work in the future, or they could be useful in other applications.

\section{MOYAL BASES AS THE $\boldsymbol{j}=0$ REPRESENTATION OF SL $(2, R)$ FOR $L_{0}, L_{ \pm 1}$}

In this section we will study certain properties of the $j$ $=0$ representation of $\operatorname{SL}(2, R)$ in relation to the continuous and discrete Moyal bases. The connection of $\operatorname{SL}(2, R)$ generated by $L_{0}, L_{ \pm 1}$ and the continuous basis is known through the work in $[21,16]$. The relevance of the $j=0$ representation is emphasized in [24]. Here we will study the $j=0$ representation with a different technique, mainly by focusing on the exponentiated group element $e^{-\tau L_{0}}$. We will extract some of the relevant properties of the $j=0$ representation that are needed in this paper. There are overlaps between our results and those in related papers [16,24-28]. We will see that all the computations in the previous sections, and similarly more general computations, amount to various matrix elements of functions of $L_{0}, L_{ \pm 1}$ in the special $j=0$ representation of $\operatorname{SL}(2, R)$.

Let us first recall why we must focus on the $j=0$ representation. The Virasoro operators $L_{0}, L_{ \pm 1}$ are the generators of the $\operatorname{SL}(2, R)$ transformations on the open string basis $X(\tau, \sigma)$. It is well known that this representation (in terms of differential operators) forms the zero Casimir representation of $\operatorname{SL}(2, R)$. Thus the oscillator basis labeled by the integers $n=1,2,3, \ldots$ is simply the case of the discrete series of $\operatorname{SL}(2, R)$ labeled as $|j, m\rangle, m=j+1, j+2, j+3, \ldots$, for $j$ $=0$.

We will use the Hermitian combinations $Q_{1}=\frac{1}{2}\left(L_{1}\right.$ $\left.+L_{-1}\right)$ and $Q_{2}=(i / 2)\left(L_{1}-L_{-1}\right)$ that form the $\operatorname{SL}(2, R)$ Lie algebra as follows $\left[Q_{1}, Q_{2}\right]=-i L_{0}, \quad\left[L_{0}, Q_{1}\right]=i Q_{2}$, $\left[L_{0}, Q_{2}\right]=-i Q_{1}$.

Thus, the odd or even discrete bases in MSFT correspond to the eigenstates of the Virasoro operator $L_{0}|n\rangle=n|n\rangle, n$ $\geqslant 1$, with $n=o, e$. The continuous basis is given by the eigenstate of the Virasoro operator $Q_{1}=\frac{1}{2}\left(L_{1}+L_{-1}\right)$ satisfying $Q_{1}|\kappa\rangle=\kappa / 2|\kappa\rangle$. The change of basis is given by the functions $\langle o \mid \kappa\rangle \equiv(1 / \sqrt{2}) V_{o}(\kappa)$ and $\langle e \mid \kappa\rangle=(1 / \sqrt{2}) V_{e}(\kappa)$. The eigenvalue of $Q_{1}$ can have any sign, but in MSFT it is possible to make transformations to the range $\kappa \geqslant 0$ thanks to the symmetry property $V_{o}(-\kappa)=V_{o}(\kappa)$ and $V_{e}(-\kappa)$ $=-V_{e}(\kappa)$ of these functions. We consider these as orthogonal matrices $V_{o}, V_{e}$ with matrix elements $\left(V_{o}\right)_{o \kappa} \equiv V_{o}(\kappa)$ 
and $\left(V_{e}\right)_{e \kappa} \equiv V_{e}(\kappa)$, for $\kappa \geqslant 0$, which satisfy orthogonality relations

$$
\begin{aligned}
& \left(V_{o} \bar{V}_{o}\right)_{o_{1} o_{2}}=\delta_{o_{1} o_{2}},\left(V_{e} \bar{V}_{e}\right)_{e_{1} e_{2}}=\delta_{e_{1} e_{2}}, \\
& \left(\bar{V}_{o} V_{o}\right)_{\kappa \kappa^{\prime}}=\delta^{(o)}\left(\kappa-\kappa^{\prime}\right),\left(\bar{V}_{e} V_{e}\right)_{\kappa \kappa^{\prime}}=\delta^{(e)}\left(\kappa-\kappa^{\prime}\right),
\end{aligned}
$$

where a bar on a matrix means the transpose of the matrix. Here $\delta^{(o, e)}\left(\kappa-\kappa^{\prime}\right)$ is basically the usual Dirac delta function, except for some extra care when $\kappa, \kappa^{\prime}$ are both close to 0 (see below), while their sum yields the usual delta function $\delta^{(o)}\left(\kappa-\kappa^{\prime}\right)+\delta^{(e)}\left(\kappa-\kappa^{\prime}\right)=2 \delta\left(\kappa-\kappa^{\prime}\right)$. These are equivalent to the orthogonality and completeness relations of the discrete and continuous bases

$$
\begin{aligned}
& \left\langle n \mid n^{\prime}\right\rangle=\delta_{n n^{\prime}}, \quad\left\langle\kappa \mid \kappa^{\prime}\right\rangle=\delta\left(\kappa-\kappa^{\prime}\right), \\
& \sum_{n=1}^{\infty}|n\rangle\left\langle n\left|=1, \quad \int_{-\infty}^{\infty} d \kappa\right| \kappa\right\rangle\langle\kappa|=1 .
\end{aligned}
$$

With the normalization given above the functions $V_{o}(\kappa), V_{e}(\kappa)$ are identical to the functions $\sqrt{2} v_{o}(\kappa), \sqrt{2} v_{e}(\kappa)$ in $[16,21]$, including an overall normalization of $\sqrt{2}$ (consistent with counterpart orthogonal transformations in the discrete basis as explained in [11]). They are given by the following generating functions (in the range $\kappa \geqslant 0)$

$$
\begin{aligned}
\sum_{o} \frac{V_{o}(\kappa)(\tan z)^{o}}{\sqrt{o}} & =\frac{\sinh (\kappa z)}{\sqrt{\kappa \sinh \left(\frac{\pi \kappa}{2}\right)}}, \\
\sum_{e} \frac{V_{e}(\kappa)(\tan z)^{e}}{\sqrt{e}} & =\frac{\cosh (\kappa z)-1}{\sqrt{\kappa \sinh \left(\frac{\pi \kappa}{2}\right)}} .
\end{aligned}
$$

To define the functions for negative values of $\kappa$ the right hand side of these equations should be multiplied by the sign function $\varepsilon(\kappa)$ for consistency with the symmetry properties of $V_{o}(-\kappa)=V_{o}(\kappa)$ and $V_{e}(-\kappa)=-V_{o}(\kappa)$.

A fundamental quantity in MSFT is the matrix $T_{e o}$ [9]. In computations $T$ usually appears in the form $t_{e o}$ as given in Eq. (4.3). This matrix is diagonalized [16] by $t_{e o}$ $=\int_{0}^{\infty} d \kappa V_{e}(\kappa) \tau(\kappa) V_{o}(\kappa) \quad$ with the eigenvalues $\tau(\kappa)$ $=\tanh (\pi \kappa / 4)$. To understand this matrix we introduce the notion of the operator $t$. Then $t_{e o}$ can be recognized to be just the matrix elements of the operator $t$ in the discrete basis

$$
t=\tanh \frac{\pi Q_{1}}{2}=\frac{1-e^{-\pi Q_{1}}}{1+e^{-\pi Q_{1}}}, \quad t_{e o}=\langle e|t| o\rangle .
$$

This is proven by introducing identity in the $\kappa$ basis and writing

$$
\begin{aligned}
t_{e o} & =\langle e|t| o\rangle=\int_{-\infty}^{\infty} d \kappa\langle e|t| \kappa\rangle\langle\kappa \mid o\rangle \\
& =\int_{-\infty}^{\infty} d \kappa \frac{1}{\sqrt{2}} V_{o}(\kappa) \tanh \frac{\pi \kappa}{4} \frac{1}{\sqrt{2}} V_{e}(\kappa) \\
& =\int_{0}^{\infty} d \kappa V_{o}(\kappa) \tanh \frac{\pi \kappa}{4} V_{e}(\kappa),
\end{aligned}
$$

where we have used the symmetry of the functions in going from the second line to the third. By the same argument we can show $t_{e e^{\prime}}=0$ and $t_{o o^{\prime}}=0$. So the matrix representation of the operator $t$ is block-off-diagonal in the even or odd basis.

Now we note the special role of the $\kappa=0$ state and its relation to $v_{o}$. The vector $v_{o}$ is a crucial state in MSFT as seen in the calculations in this paper. Its special role in relation to anomalies first emerged in $[9,10]$ as a state closely related to the midpoint of the string. From the generating functions in Eq. (5.3) it is seen that at $\kappa=0$ the even functions vanish $V_{e}(0)=0$, while the odd ones $V_{o}(0)$ are finite,

$$
V_{e}(0)=0, \quad V_{o}(0)=\frac{1}{2} \sqrt{\pi o} v_{o}
$$

where $v_{o}$ is given in Eq. (4.3). It is a normalized vector $\Sigma_{o \geqslant 1} \bar{v}_{o} v_{o}=1$ that is a zero mode of the matrix $T: \Sigma_{o \geqslant 1} T_{e o} v_{o}=0$. Equivalently, $V_{o}(0)$ is the zero mode of the matrix $t_{e o}$. This is easily seen from the expression Eq. (5.4) for the $t$ operator acting on the zero eigenstate in the kappa basis $Q_{1}|0\rangle=0$

$$
t_{e o} V_{o}(0)=0 \leftrightarrow e^{-\pi Q_{1}}|0\rangle=|0\rangle .
$$

Note that we can write $v_{o}$ in terms of the $\kappa=0$ state as follows:

$$
\left(\kappa_{o}^{-1 / 2} v\right)_{o}=(2 / \sqrt{\pi})\left\langle o\left|\frac{1}{L_{0}}\right| 0\right\rangle .
$$

We can use the above interpretation of the operator $t$ and and its zero mode $v_{o}$ to give an $\operatorname{SL}(2, R)$ operator representation of the computations we performed in the previous section. In particular, we see that $b_{o}, b_{e}$ of Eqs. (3.8) can now be written as matrix elements in the $j=0$ representation of $\operatorname{SL}(2, R)$

$$
b_{o}=\frac{2}{\sqrt{\pi}}\left\langle o\left|\frac{2}{3+t^{2}} \frac{1}{L_{0}}\right| 0\right\rangle, \quad b_{e}=\frac{2}{\sqrt{\pi}}\left\langle e\left|\frac{2 t}{3+t^{2}} \frac{1}{L_{0}}\right| 0\right\rangle \text {. }
$$

Note that $\left\langle e\left|\left[2 /\left(3+t^{2}\right)\right]\left(1 / L_{0}\right)\right| 0\right\rangle=0$ since $\langle e \mid 0\rangle=0$. Hence we can remove the bras and write the states

$$
\left|b_{o}\right\rangle=\frac{2}{\sqrt{\pi}} \frac{2}{3+t^{2}} \frac{1}{L_{0}}|0\rangle,\left|b_{e}\right\rangle=\frac{2}{\sqrt{\pi}} \frac{2 t}{3+t^{2}} \frac{1}{L_{0}}|0\rangle
$$


where now the $o, e$ indices may be interpreted as an odd or even number of powers of the operator $t$. Similarly, the quantities $\alpha(\tau), \beta(\tau), \gamma(\tau)$ that appear in the 4-tachyon amplitude in Eqs. (3.2)-(3.4) become expectation values in the $\kappa=0$ states. For example

$$
\begin{aligned}
\beta(\tau)= & \bar{b} P^{(o)} e^{-\tau \kappa}\left(1-M e^{-\tau \kappa} M e^{-\tau \kappa}\right)^{-1} P^{(o)} b, \\
= & \frac{4}{\pi}\langle 0| \frac{1}{L_{0}} \frac{2}{3+t^{2}} e^{-\tau L_{0}} \\
& \times\left(1-\frac{1-t^{2}}{3+t^{2}} e^{-\tau L_{0}} \frac{1-t^{2}}{3+t^{2}} e^{-\tau L_{0}}\right)^{-1} \\
& \times \frac{2}{3+t^{2}} \frac{1}{L_{0}}|0\rangle .
\end{aligned}
$$

Once we notice these forms we are tempted to use the commutation rules of the operators of $\operatorname{SL}(2, R)$ to simplify and compute these quantities. In particular note that $e^{-\omega Q_{1}} L_{0} e^{\omega Q_{1}}=L_{0} \cos \omega+i Q_{2} \sin \omega$ gives the following interesting properties of $t$ and $e^{-\pi Q_{1}}$ for $\omega=\pi$

$$
\begin{aligned}
e^{-\pi Q_{1}} L_{0} & =-L_{0} e^{-\pi Q_{1}}, t L_{0}=L_{0} t^{-1}, \\
\frac{1}{L_{0}} t & =t^{-1} \frac{1}{L_{0}}, t \frac{1}{L_{0}}=\frac{1}{L_{0}} t^{-1}, \text { etc. }
\end{aligned}
$$

Using these it appears as if we may perform a number of simplifications very efficiently. Alas, there is the problem of anomalies precisely because of the existence of the $\kappa=0$ state on which $t$ vanishes. So its inverse cannot be used in a cavalier fashion. In fact not all of the above formulas are necessarily valid in general since they depend on which state they are applied. When we introduce a complete set of intermediate states between operators, the $\kappa=0$ state must appear, causing problems. One can easily generate inconsistent results from the same formula with naive manipulations of $t$. Examples of inconsistencies can be easily constructed by taking matrix elements and using naively the formulas above on the $\kappa=0$ state. The type of formal manipulations suggested above need to be justified at every step or otherwise replaced by the correct result.

Thus, practical computations in string field theory require a regulator at $\kappa=0$ because the matrix $t_{e o}$ has certain associativity anomalies that come from the zero modes reviewed above. This is precisely the zero mode of $T_{e o}$ and the associativity anomaly issue which are intimately related to midpoint issues in string field theory as first understood in [10].

A regulated version of the matrix $t_{e o}$ with many nice mathematical properties was introduced in $[10,11,13]$. The regulated matrices retain the properties of the operator $t$ that are always valid [see Eq. (2.15) in [11]]. In the regularized theory, the continuous functions $V_{o}(\kappa), V_{e}(\kappa)$ can be thought of as the large $N$ limit of $N \times N$ matrices $\left(V_{o}\right)_{o k},\left(V_{e}\right)_{e k}$ with discrete values of $k$ which label the $N$ eigenvalues $\tau_{k}$ of the regularized $N \times N$ matrix $t_{e o}$
$=V_{e} \tau \bar{V}_{o}$. The large $N$ limits of the matrix elements $\left(V_{o}\right)_{o k},\left(V_{e}\right)_{e k}$ become the functions $V_{o}(\kappa), V_{e}(\kappa)$, and the discrete eigenvalues $\tau_{k}$ become $\tau_{k} \rightarrow \tau(\kappa)=\tanh (\pi \kappa / 4)$. In the limit $N \rightarrow \infty$, summation over discrete $k$ is replaced by $\int_{0}^{\infty} d \kappa$ and the discrete delta function $\delta_{k k^{\prime}}$ is replaced by the continuous delta functions $\delta^{(o, e)}\left(\kappa-\kappa^{\prime}\right)$ with non-negative $\kappa, \kappa^{\prime} \geqslant 0$, but with some extra care when $\kappa, \kappa^{\prime}$ are both close to zero, as we will see below.

In this section we try to relate the large $N$ limit of the regulated theory in the discrete basis to the regulated continuous kappa basis, and from this extract computational methods that take advantage correctly of both the discrete and continuous bases. For this purpose we will study several quantities by taking advantage of the $\operatorname{SL}(2, R)$ group generated by the Virasoro operators $L_{0}, L_{1}, L_{-1}$.

\section{A. Propagator $\left\langle\kappa\left|e^{-\tau L_{0}}\right| \boldsymbol{\kappa}^{\prime}\right\rangle$}

The quantity $D_{\kappa \kappa^{\prime}}(\tau)=\left\langle\kappa\left|e^{-\tau L_{0}}\right| \kappa^{\prime}\right\rangle$ is a representation of a group element of $\mathrm{SL}(2, R)$ with Lie generators $\left(L_{0}, L_{ \pm 1}=Q_{1} \mp i Q_{2}\right)$, since $|\kappa\rangle$ is the basis that diagonalizes the generator $Q_{1}=\frac{1}{2}\left(L_{1}+L_{-1}\right)$, with $Q_{1}|\kappa\rangle=(\kappa / 2)|\kappa\rangle$. Its integral and derivatives are quantities of interest in various explicit computations in MSFT

$$
\begin{aligned}
\left\langle\kappa\left|\frac{1}{L_{0}}\right| \kappa^{\prime}\right\rangle & =\int_{0}^{\infty} d \tau D_{\kappa \kappa^{\prime}}(\tau), \\
\left\langle\kappa\left|L_{0}\right| \kappa^{\prime}\right\rangle & =\left[-\partial_{\tau} D_{\kappa \kappa^{\prime}}(\tau)\right]_{\tau=0} .
\end{aligned}
$$

In this section we compute $D_{\kappa \kappa^{\prime}}(\tau)$ as a group element of $\operatorname{SL}(2, R)$ in the special representation $j=0$. Of course, in the discrete basis $e^{-\tau L_{0}}$ is diagonal, therefore we expect

$$
\begin{aligned}
D_{\kappa \kappa^{\prime}}(\tau) & =\left\langle\kappa\left|e^{-\tau L_{0}}\left(\sum_{e}|e\rangle\left\langle e\left|+\sum_{o}\right| o\right\rangle\langle o|\right)\right| \kappa^{\prime}\right\rangle \\
& =\frac{1}{2}\left(\bar{V}_{o} e^{-\kappa_{o} \tau} V_{o}\right)_{\kappa \kappa^{\prime}}+\frac{1}{2}\left(\bar{V}_{e} e^{-\kappa_{e} \tau} V_{e}\right)_{\kappa \kappa^{\prime}} .
\end{aligned}
$$

where $\kappa_{e}, \kappa_{o}$ are the diagonal matrices $\kappa_{e}$ $=\operatorname{diag}(2,4,6, \ldots)$ and $\kappa_{o}=\operatorname{diag}(1,3,5, \ldots)$, and the $V_{o}, V_{e}$ are treated as matrices with matrix elements that are the functions $\left(V_{o}\right)_{o \kappa}=V_{o}(\kappa)$, etc.

Following the methods in [22], Chap. 7.4 [leading to Eq. (7.65)], we can derive a differential equation for $D_{\kappa \kappa^{\prime}}(\tau)$ as follows. We use the fact that the eigenvalue of the quadratic Casimir operator vanishes for the states $|\kappa\rangle$ to write

$$
\left\langle\kappa\left|e^{-\tau L_{0}}\left(\left(L_{0}^{2}-Q_{1}^{2}-Q_{2}^{2}\right)\right)\right| \kappa^{\prime}\right\rangle=0 .
$$

The first two terms can be rewritten as follows:

$$
\begin{gathered}
\left\langle\kappa\left|e^{-\tau L_{0}} L_{0}^{2}\right| \kappa^{\prime}\right\rangle=\partial_{\tau}^{2} D_{\kappa \kappa^{\prime}}(\tau), \\
\left\langle\kappa\left|e^{-\tau L_{0}}\left(-Q_{1}^{2}\right)\right| \kappa^{\prime}\right\rangle=-\left(\frac{\kappa^{\prime}}{2}\right)^{2} D_{\kappa \kappa^{\prime}}(\tau) .
\end{gathered}
$$


The third term $\left\langle\kappa\left|e^{-\tau L_{0}}\left(-Q_{2}^{2}\right)\right| \kappa^{\prime}\right\rangle$ can also be computed by noting the following property, $e^{\tau L_{0}} Q_{1} e^{-\tau L_{0}}=Q_{1} \cosh \tau$ $+i Q_{2} \sinh \tau$, which can be rewritten as

$$
e^{-\tau L_{0}} i Q_{2}=\frac{1}{\sinh \tau} Q_{1} e^{-\tau L_{0}}-\frac{\cosh \tau}{\sinh \tau} e^{-\tau L_{0}} Q_{1} .
$$

Multiplying Eq. (5.21) with $i Q_{2}$, using $Q_{1} Q_{2}=Q_{2} Q_{1}$ $-i L_{0}$, and using Eq. (5.21) again, gives

$$
\begin{aligned}
-e^{-\tau L_{0}} Q_{2}^{2}= & \frac{i}{\sinh \tau} Q_{1}\left(e^{-\tau L_{0}} Q_{2}\right)-i \frac{\cosh \tau}{\sinh \tau}\left(e^{-\tau L_{0}} Q_{2}\right) Q_{1} \\
& -\frac{\cosh \tau}{\sinh \tau} e^{-\tau L_{0}} L_{0} \\
= & \frac{1}{\sinh ^{2} \tau} Q_{1}^{2} e^{-\tau L_{0}}-2 \frac{\cosh \tau}{\sinh ^{2} \tau} Q_{1} e^{-\tau L_{0}} Q_{1} \\
& +\frac{\cosh ^{2} \tau}{\sinh ^{2} \tau} e^{-\tau L_{0}} Q_{1}^{2}+\frac{\cosh \tau}{\sinh \tau} \partial_{\tau} e^{-\tau L_{0}}
\end{aligned}
$$

The matrix elements of this relation are easily evaluated in terms of $D_{\kappa \kappa^{\prime}}(\tau)$. Combining the three terms in Eq. (5.19) we obtain the differential equation satisfied by $D_{\kappa \kappa^{\prime}}(\tau)$

$$
\left(\partial_{\tau}^{2}+\frac{\cosh \tau}{\sinh \tau} \partial_{\tau}+\frac{\kappa^{2}+\left(\kappa^{\prime}\right)^{2}-2 \kappa \kappa^{\prime} \cosh \tau}{4 \sinh ^{2} \tau}\right) D_{\kappa \kappa^{\prime}}(\tau)=0 .
$$

By taking $D_{\kappa \kappa^{\prime}}(\tau)=z(1-z)^{-i\left(\kappa+\kappa^{\prime}\right) / 4} F(z)$, with

$$
z=-\left(\sinh \frac{\tau}{2}\right)^{-2}=-4 e^{-\tau}\left(1-e^{-\tau}\right)^{-2},
$$

this differential equation becomes the hypergeometric differential equation for $F(z)$

$$
\left(\left(\frac{\partial}{\partial z}\right)^{2}+\frac{c-(1+a+b) z}{z(1-z)} \frac{\partial}{\partial z}-\frac{a b}{z(1-z)}\right) F(z)=0,
$$

with $F(a, b ; c ; z) \sim$ hypergeom $([a, b],[c], z)$, and

$$
a=1-i \frac{\kappa}{2}, \quad b=1-i \frac{\kappa^{\prime}}{2}, \quad c=2 .
$$

We need the solution that satisfies the boundary conditions that follow from Eq. (5.18)

$$
\begin{gathered}
D_{\kappa \kappa^{\prime}}(\tau) \underset{\tau \rightarrow \infty}{\longrightarrow} \frac{1}{2} V_{1}(\kappa) V_{1}\left(\kappa^{\prime}\right) e^{-\tau}, \\
D_{\kappa \kappa^{\prime}}(\tau) \underset{\tau \rightarrow 0}{\longrightarrow} \delta\left(\kappa-\kappa^{\prime}\right),
\end{gathered}
$$

where $V_{1}(\kappa)=\sqrt{\kappa / \sinh (\pi \kappa / 2)}$, as obtained from Eq. (5.3). Therefore, the desired solution is given by

$$
\begin{aligned}
D_{\kappa \kappa^{\prime}}(\tau)= & \sqrt{\frac{\kappa \kappa^{\prime}}{\sinh \frac{\pi \kappa}{2} \sinh \frac{\pi \kappa^{\prime}}{2}}} \frac{e^{-\tau}}{2\left(1-e^{-\tau}\right)^{2}}\left(\frac{1-e^{-\tau}}{1+e^{-\tau}}\right)^{i \kappa / 2+i \kappa^{\prime} / 2} \\
& \times F\left(1-\frac{i \kappa}{2}, 1-\frac{i \kappa^{\prime}}{2} ; 2 ; \frac{-4 e^{-\tau}}{\left(1-e^{-\tau}\right)^{2}}\right) .
\end{aligned}
$$

By using the linear identity $F(a, b ; c ; z)=(1-z)^{c-a-b} F(c-a, c-b ; c ; z)$, we see that the expression for $D_{\kappa \kappa^{\prime}}(\tau)$ is real. At $\tau=\infty$ the boundary condition is satisfied since $F(a, b ; c ; 0)=1$. For small $\tau \sim 0$, and any $\kappa, \kappa^{\prime}$, we use the asymptotic behavior of the hypergeometric function [23] to write

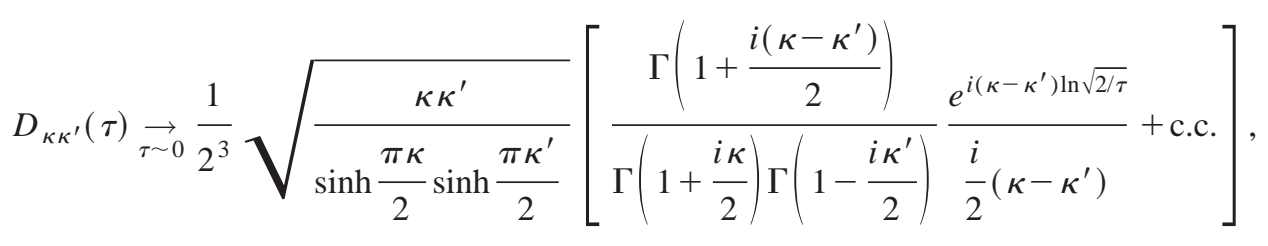

where c.c. is the complex conjugate. For small $\left(\kappa^{\prime}-\kappa\right) \sim 0$ and small $\tau \sim 0$ we can write $\Gamma(1+i \kappa / 2) \Gamma(1-i \kappa / 2)=(\pi \kappa / 2)$ $\times(\sinh \pi \kappa / 2)^{-1}$, so that the singular behavior of $D_{\kappa \kappa^{\prime}}(\tau)$, consistent with the delta function, becomes evident as follows: 


$$
\begin{aligned}
{\left[D_{\kappa \kappa^{\prime}}(\tau)\right]_{\tau \sim 0} \simeq } & \frac{\sin \left(\left(\kappa-\kappa^{\prime}\right) \ln \sqrt{\frac{2}{\tau}}\right)}{\pi\left(\kappa-\kappa^{\prime}\right)} \\
& =\frac{1}{2 \pi} \int_{-\ln \sqrt{2 / \tau}}^{\ln \sqrt{2 / \tau}} d u e^{i u\left(\kappa-\kappa^{\prime}\right)} \underset{\tau \rightarrow 0}{=} \delta\left(\kappa-\kappa^{\prime}\right) .
\end{aligned}
$$

We also record here the special values for $\kappa, \kappa^{\prime}=0$, that follow from $F(a, 1 ; 2 ; z)=\left[(1-z)^{-a} z-(1-z)^{-a}+1\right] / z(1$ $-a)$ at any $\tau$

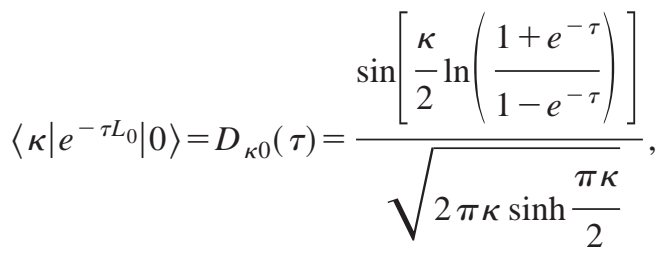

$$
\begin{aligned}
& \left\langle 0\left|e^{-\tau L_{0}}\right| 0\right\rangle=D_{00}(\tau)=\frac{1}{2 \pi} \ln \left(\frac{1+e^{-\tau}}{1-e^{-\tau}}\right),
\end{aligned}
$$

where the second line follows by setting $\kappa=0$ in the first line.

The integrals of these quantities give $\left\langle\kappa\left|1 / L_{0}\right| 0\right\rangle$ and $\left\langle 0\left|1 / L_{0}\right| 0\right\rangle$

$$
\begin{aligned}
& \left\langle\kappa\left|\frac{1}{L_{0}}\right| 0\right\rangle=\int_{0}^{\infty} d \tau D_{\kappa 0}(\tau)=\left(\frac{\pi \tanh \frac{\pi \kappa}{4}}{16 \kappa \cosh ^{2} \frac{\pi \kappa}{4}}\right)^{1 / 2}, \\
& \left\langle 0\left|\frac{1}{L_{0}}\right| 0\right\rangle=\frac{\pi}{8}
\end{aligned}
$$

This agrees with an independent calculation in Eq. (5.64).

The derivative near $\tau \sim 0$ gives information about matrix elements of $L_{0}$

$$
\begin{aligned}
\left\langle\kappa\left|L_{0}\right| \kappa^{\prime}\right\rangle= & {\left[-\partial_{\tau} D_{\kappa \kappa^{\prime}}(\tau)\right]_{\tau \sim 0} } \\
= & \frac{1}{2^{3}} \sqrt{\frac{\kappa \kappa^{\prime}}{\sinh \frac{\pi \kappa}{2} \sinh \frac{\pi \kappa^{\prime}}{2}}} \\
& \times\left[\frac{\Gamma\left(1+\frac{i\left(\kappa-\kappa^{\prime}\right)}{2}\right) e^{i\left(\kappa-\kappa^{\prime}\right) \ln \sqrt{2 / \tau}}}{\Gamma\left(1+\frac{i \kappa}{2}\right) \Gamma\left(1-\frac{i \kappa^{\prime}}{2}\right) \tau}+\text { c.c. }\right] .
\end{aligned}
$$

This diverges due to the factor $1 / \tau$. More simply, the quantity $\left\langle\kappa\left|L_{0}\right| 0\right\rangle$ is obtained also by studying the derivative of Eq. (5.32) at any $\tau$

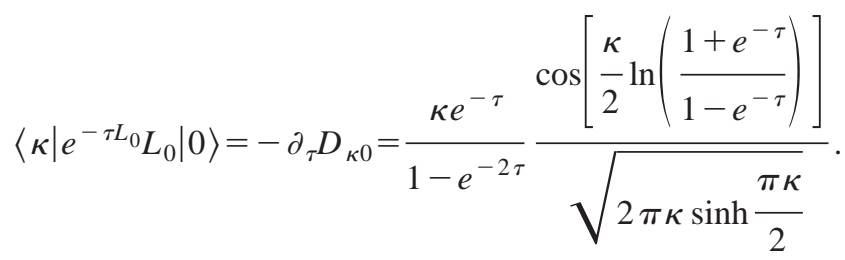

Near $\tau \sim 0$ one sees that $\left\langle\kappa\left|L_{0}\right| 0\right\rangle$ and $\left\langle 0\left|L_{0}\right| 0\right\rangle$ have a singular behavior

$$
\begin{aligned}
& \left\langle\kappa\left|L_{0}\right| 0\right\rangle \rightarrow\left(\frac{\frac{\pi \kappa}{2}}{\sinh \frac{\pi \kappa}{2}}\right)^{1 / 2} \frac{\cos \left[\frac{\kappa}{2} \ln \left(\frac{2}{\tau}\right)\right]}{2 \pi \tau}, \\
& \left\langle 0\left|L_{0}\right| 0\right\rangle \rightarrow \frac{1}{2 \pi \tau} .
\end{aligned}
$$

Let us also compute $\left\langle\kappa\left|L_{0}\right| \kappa^{\prime}\right\rangle$ for $\kappa^{\prime} \sim \kappa$ from Eq. (5.36) or from Eq. (5.31). We obtain the singular behavior

$$
\begin{aligned}
{\left[\left\langle\kappa\left|L_{0}\right| \kappa^{\prime}\right\rangle\right]_{\kappa^{\prime} \sim \kappa} } & =\left[-\partial_{\tau} D_{\kappa \kappa^{\prime}}(\tau)\right]_{\tau \sim 0, \kappa^{\prime} \sim \kappa} \\
& \approx \frac{\cos \left(\left(\kappa-\kappa^{\prime}\right) \ln \sqrt{\frac{2}{\tau}}\right)}{2 \pi \tau}
\end{aligned}
$$

which is consistent with Eq. (5.38), but now gives

$$
\left\langle\kappa\left|L_{0}\right| \kappa\right\rangle=\left[-\partial_{\tau} D_{\kappa \kappa}(\tau)\right]_{\tau \sim 0} \approx \frac{1}{2 \pi \tau}
$$

at any $\kappa$. The divergence of $\left\langle\kappa\left|L_{0}\right| \kappa^{\prime}\right\rangle$ is also discussed in [16].

The quickly oscillating factor $\exp \left(i\left(\kappa-\kappa^{\prime}\right) \ln \sqrt{2 / \tau}\right)$ in Eq. (5.36) suggests that only the neighborhood of $\kappa^{\prime} \sim \kappa$ is relevant in a steepest descent approximation when the function $\left\langle\kappa\left|L_{0}\right| \kappa^{\prime}\right\rangle$ is integrated with smooth functions $\int d \kappa \int d \kappa^{\prime} f(\kappa)\left\langle\kappa\left|L_{0}\right| \kappa^{\prime}\right\rangle g\left(\kappa^{\prime}\right) \sim(1 / 2 \pi \tau) \int d \kappa f(\kappa) g(\kappa) \int d q$ $\times \cos (q \ln \sqrt{2 / \tau})$, where we have used Eq. (5.39), and implied a cutoff in the integration in $q$ which depends on the functions $f, g$. This expression is also divergent when $\tau$ vanishes for well behaved $f, g$. This last form relates directly to the quantity $\dot{\gamma}(0)$ in Eq. (3.39) through Eqs. (4.16), (4.17) as the regulator $\tau$ is removed. We used the singular behavior $\dot{\gamma}(0)=\infty$ to determine the midrange slope (i.e. differentiability) of the off-shell factor in the 4-tachyon scattering. 


\section{Computation of $\left(\bar{V}_{e} e^{-\kappa_{e} \tau} V_{e}\right)_{\kappa \kappa^{\prime}}$ and $\left(\bar{V}_{o} e^{-\kappa_{o} \tau} V_{o}\right)_{\kappa \kappa^{\prime}}$}

To compute $\left(\bar{V}_{e} e^{-\kappa_{e} \tau} V_{e}\right)_{\kappa \kappa^{\prime}}$ and $\left(\bar{V}_{o} e^{-\kappa_{o} \tau} V_{o}\right)_{\kappa \kappa^{\prime}}$, we first recall their sum as in Eq. (5.18), and then extract the individual terms from the symmetry under reflections with respect to $\kappa$ or $\kappa^{\prime}$ :

$$
\begin{aligned}
\left(\bar{V}_{o} e^{-\kappa_{o} \tau} V_{o}\right)_{\kappa \kappa^{\prime}} & =D_{\kappa \kappa^{\prime}}(\tau)+D_{(-\kappa) \kappa^{\prime}}(\tau) \\
& =D_{\kappa \kappa^{\prime}}(\tau)+D_{\kappa\left(-\kappa^{\prime}\right)}(\tau), \\
\left(\bar{V}_{e} e^{-\kappa_{e} \tau} V_{e}\right)_{\kappa \kappa^{\prime}} & =D_{\kappa \kappa^{\prime}}(\tau)-D_{(-\kappa) \kappa^{\prime}}(\tau) \\
& =D_{\kappa \kappa^{\prime}}(\tau)-D_{\kappa\left(-\kappa^{\prime}\right)}(\tau) .
\end{aligned}
$$

where we insert our result (5.29) for $D_{\kappa \kappa^{\prime}}(\tau)$.

In particular, if we take $\kappa^{\prime}=0$ we get $\left(\bar{V}_{e} e^{-\kappa_{e} \tau} V_{e}\right)_{\kappa 0}$ $=0$, while $\left(\bar{V}_{o} e^{-\kappa_{o} \tau} V_{o}\right)_{\kappa 0}=2 D_{\kappa 0}(\tau)$, which implies

$$
\begin{aligned}
\bar{v}_{o} \kappa_{o}^{1 / 2} e^{-\kappa_{o} \tau} V_{o}(\kappa) & =\frac{2}{\sqrt{\pi}}\left(\bar{V}_{o} e^{-\kappa_{o} \tau} V_{o}\right)_{\kappa 0}=\frac{4}{\sqrt{\pi}}\left\langle 0\left|e^{-L_{0} \tau}\right| \kappa\right\rangle \\
& =\frac{4}{\sqrt{\pi}} D_{\kappa 0}(\tau) \\
& =\frac{2}{\pi} \frac{\sin \left[\frac{\kappa}{2} \ln \left(\frac{1+e^{-\tau}}{1-e^{-\tau}}\right)\right]}{\sqrt{\frac{\kappa}{2} \sinh \frac{\pi \kappa}{2}}}
\end{aligned}
$$

Taking also $\kappa=0$ gives

$$
\begin{aligned}
\bar{v}_{o} \kappa_{o} e^{-\kappa_{o} \tau} v_{o} & =\frac{4}{\pi}\left(\bar{V}_{o} e^{-\kappa_{o} \tau} V_{o}\right)_{00}=\frac{8}{\pi}\left\langle 0\left|e^{-L_{0} \tau}\right| 0\right\rangle \\
& =\frac{8}{\pi} D_{00}(\tau) \\
& =\frac{4}{\pi^{2}} \ln \left(\frac{1+e^{-\tau}}{1-e^{-\tau}}\right)
\end{aligned}
$$

We are now ready to tackle the quantity $\bar{v}_{o} \kappa_{o}^{-1 / 2} V_{o}(\kappa)$ $=(2 / \sqrt{\pi}) \Sigma_{o>0} \bar{V}_{o}(0)(1 / o) V_{o}(\kappa)$ which is needed in explicit computations in MSFT, as in Eq. (4.7) and the equations that follow it. Since $V_{e}(0)=0$, only the odd $V_{o}(0)$ contribute when the identity $1=\Sigma_{n}|n\rangle\langle n|$ is inserted in $\left\langle\kappa\left|\left(1 / L_{0}\right) 1\right| 0\right\rangle$

$$
\frac{1}{2}\left(\bar{V}_{o} \frac{1}{o} V_{o}\right)_{\kappa 0}=\left\langle\kappa\left|\frac{1}{L_{0}}\right| 0\right\rangle=\left(\frac{\pi \tanh \frac{\pi \kappa}{4}}{16 \kappa \cosh ^{2} \frac{\pi \kappa}{4}}\right)^{1 / 2},
$$

where we used the result in Eq. (5.34). From this we obtain immediately

$$
\bar{v}_{o} \kappa_{o}^{-1 / 2} V_{o}(\kappa)=\frac{4}{\sqrt{\pi}}\left(\frac{1}{2} \bar{V}_{o} \frac{1}{o} V_{o}\right)_{\kappa 0}=\left(\frac{\tanh \frac{\pi \kappa}{4}}{\kappa \cosh ^{2} \frac{\pi \kappa}{4}}\right)^{1 / 2} .
$$

This result is used to relate regulated discrete basis computations of the type $\bar{v}_{o} \kappa_{o}^{-1 / 2}(f(\bar{t} t))_{o o^{\prime}} \kappa_{o^{\prime}}^{-1 / 2} v_{o^{\prime}}$ in the large $N$ limit to kappa basis computations as shown in the previous section.

\section{Computation of $\rho(\kappa)=\langle\kappa \mid \kappa\rangle$}

We have argued in Eq. (5.31) that near $\tau=0$ and $\kappa^{\prime} \sim \kappa$ we obtain $D_{\kappa \kappa^{\prime}}(0)=\left\langle\kappa \mid \kappa^{\prime}\right\rangle=\delta\left(\kappa-\kappa^{\prime}\right)$. The quantity $\rho(\kappa)=\langle\kappa \mid \kappa\rangle$ appears in the evaluation of determinants as in

$$
\operatorname{det}(f(t \bar{t}))=\exp \left[\int_{0}^{\infty} d \kappa \rho(\kappa) \ln f\left(\tanh ^{2} \frac{\pi \kappa}{4}\right)\right] .
$$

Since we expect a singular behavior, $\rho(\kappa) \sim \delta(0)$, we would like to perform such computations with a finite number of modes $2 N$ and send $N$ to infinity at the end of the computation. Thus, on the left side we have a finite determinant that depends on $N$, which in principle can be computed in the regulated basis, at least numerically. To perform such careful computations analytically by taking advantage of the $\kappa$ basis, we define $\rho(\kappa)=\langle\kappa \mid \kappa\rangle$ carefully at $\kappa^{\prime}=\kappa$ for small but finite $\tau$, and compare to a similar calculation with the cutoff in terms of the number of modes $2 N$. Thus, for $\kappa^{\prime}= \pm \kappa$ the cases $D_{\kappa \kappa}(\tau), D_{\kappa(-\kappa)}(\tau)$, as $\tau \rightarrow 0$, are used to compute the quantity $\rho(\kappa, \tau) \simeq\langle\kappa \mid \kappa\rangle$ as follows. From Eq. (5.30) and from the asymptotic behavior of the hypergeometric function at large $(-z)$

$$
\begin{aligned}
F(a, a, c ; z) \rightarrow & \frac{\Gamma(c)(-z)^{-a}}{\Gamma(a) \Gamma(c-a)}(\ln (-z)+2 \psi(1)-\psi(a) \\
& -\psi(c-a))
\end{aligned}
$$

we obtain

$$
\begin{aligned}
D_{\kappa \kappa}(\tau) \underset{\tau \sim 0}{\rightarrow}= & \frac{1}{4 \pi}\left(\ln \left(\frac{2}{\tau}\right)^{2}+2 \psi(1)-\psi\left(1-\frac{i \kappa}{2}\right)\right. \\
& \left.-\psi\left(1+\frac{i \kappa}{2}\right)\right)+O\left(\tau^{2}\right) \\
D_{\kappa(-\kappa)}(\tau) \underset{\tau \sim 0}{\rightarrow}= & \frac{1}{2^{3} i \sinh \frac{\pi \kappa}{2}}\left(\frac{\Gamma(1+i \kappa)}{\Gamma^{2}\left(1+\frac{i \kappa}{2}\right)} e^{i \kappa \ln (2 / \tau)}\right. \\
& \left.-\frac{\Gamma(1-i \kappa)}{\Gamma^{2}\left(1-\frac{i \kappa}{2}\right)} e^{-i \kappa \ln (2 / \tau)}\right)+O\left(\tau^{2}\right) .
\end{aligned}
$$


Note the tricky behavior near $\kappa=0$ : as long as $\kappa$ is not close to zero, the quantity $\lim _{\tau \rightarrow 0} D_{\kappa(-\kappa)}(\tau)$ oscillates rapidly and becomes negligible as a distribution compared to $\lim _{\tau \rightarrow 0} D_{\kappa \kappa}(\tau)$. However, near $\kappa=0$ we get

$$
\begin{aligned}
D_{(-\kappa) \kappa}(\tau) \underset{\tau \sim 0, \kappa \sim 0}{\rightarrow}= & \frac{1}{4 \pi} \ln \left(\frac{2}{\tau}\right)^{2} \\
& +\kappa^{2}\left(-\frac{\pi \ln \frac{2}{\tau}}{24}-\frac{\ln ^{3} \frac{2}{\tau}}{12 \pi}+0.04783\right) \\
& +O\left(\kappa^{4}\right) .
\end{aligned}
$$

Hence, if $\kappa \neq 0$ not small, and $\tau$ is small, we neglect $D_{(-\kappa) \kappa}(\tau)$ and get $\left(\bar{V}_{o} e^{-\tau o} V_{o}\right)_{\kappa \kappa} \sim\left(\bar{V}_{e} e^{-\tau e} V_{e}\right)_{\kappa \kappa} \sim D_{\kappa \kappa}(\tau)$ $\sim \rho(\kappa, \tau)$ with

$\rho(\kappa, \tau)=\frac{1}{4 \pi}\left(\ln \left(\frac{2}{\tau}\right)^{2}+2 \psi(1)-\psi\left(1-\frac{i \kappa}{2}\right)-\psi\left(1+\frac{i \kappa}{2}\right)\right)$.

But, close to $\kappa=0$, because of Eq. (5.53), the behavior is

$$
\begin{aligned}
& \kappa, \tau \sim 0:\left(\bar{V}_{o} e^{-\tau o} V_{o}\right)_{00}=2[\rho(0, \tau)]_{\tau \sim 0}=\frac{2}{4 \pi} \ln \left(\frac{2}{\tau}\right)^{2}, \\
&\left(\bar{V}_{e} e^{-\tau e} V_{e}\right)_{00}=0 .
\end{aligned}
$$

The factor of 2 computed here is important to establish consistency between the regulators in the discrete and continuous bases.

We concentrate on $\rho(\kappa)$ for the general $\kappa$. We already understand its dependence on $\kappa$ explicitly, and therefore write it in the form

$$
\rho(\kappa)=\rho(0)+\frac{1}{4 \pi}\left(2 \psi(1)-\psi\left(1-\frac{i \kappa}{2}\right)-\psi\left(1+\frac{i \kappa}{2}\right)\right) .
$$

We know $\rho(0)$ is divergent, and is given by

$$
\begin{aligned}
\rho(0) & =\frac{1}{2}\left(\bar{V}_{o} V_{o}\right)_{00}=\langle 0 \mid 0\rangle=\frac{\pi}{8}\left(v_{o} \kappa_{o} v_{o}\right)_{\text {regulated }} \\
& =\frac{1}{2 \pi}\left(\ln (2 N)+\Delta^{\prime}\right)
\end{aligned}
$$

where we used a finite number of modes $2 N$ as the regulator and the constant $\Delta^{\prime}$ depends on further details of the regulator. Therefore the full regulated $\rho(\kappa)$ is given by

$$
\rho(\kappa)=\frac{1}{2 \pi}(\ln (2 N)+\Delta)-\frac{1}{4 \pi}\left(\psi\left(1-\frac{i \kappa}{2}\right)+\psi\left(1+\frac{i \kappa}{2}\right)\right)
$$

where $\Delta$ is a constant. Note that this constant can be absorbed into a redefinition of $N$ by writing $\ln (2 N)+\Delta$ $=\ln \left(2 N e^{\Delta}\right)$. We will further determine $\Delta=0$ by comparing to a particular regulator in the discrete basis of MSFT in which we keep the frequencies as simple as possible, namely $\kappa_{n}$ $=n$. In principle it is a dangerous business to compare diverging quantities when using different regulators. However, as we have already mentioned, since the $N$ dependence will drop out in certain computations in $d=26$ then $\Delta$ will also drop out, and hence its value may not be important.

\section{B. Exact analytic tests}

As tests of the formulas we derived above in the kappa basis we compare to exact computations in the regulated ${ }^{6}$ discrete basis, in the limit $N \rightarrow \infty$. The first case is $\bar{v} \kappa_{o}^{-1 / 2} 1 \kappa_{o}^{-1 / 2} v$ where we compare Eq. (4.13) versus using directly the $v_{o}$ in (4.3) or the regulated one in footnote 6 . The two ways of computing give the same answer:

$$
\begin{aligned}
& \left(\bar{v} \kappa_{o}^{-1 / 2} 1 \kappa_{o}^{-1 / 2} v\right)_{\text {discrete }} \\
& \quad=\frac{8}{\pi^{2}} \sum_{n=1}^{\infty} \frac{1}{(2 n-1)^{3}}+O\left((\ln N) / N^{2}\right)
\end{aligned}
$$

$$
\begin{aligned}
\underset{N \rightarrow \infty}{\rightarrow} \frac{7 \zeta(3)}{\pi^{2}} & =0.85256 \\
\left(\bar{v} \kappa_{o}^{-1 / 2} 1 \kappa_{o}^{-1 / 2} v\right)_{k a p p a} & =\int_{0}^{\infty} d \kappa \frac{\tanh \frac{\pi \kappa}{4}}{\kappa\left(\cosh \frac{\pi \kappa}{4}\right)^{2}} \times 1 \\
& =0.85256 .
\end{aligned}
$$

The second case is $\operatorname{det}(\bar{t} t)=\exp \left[\int_{0}^{\infty} d \kappa \rho(\kappa)\right.$ $\left.\times \ln \left(f\left[\tanh ^{2}(\pi \kappa / 4)\right]\right)\right]$ where we use $\rho(\kappa)$ in Eq. (4.24), while the exact computation in the regulated discrete basis was given in $[10,11]$ for any $N$ as

${ }^{6}$ To avoid anomalies we use the standard regularization of MSFT $[10,11,13]$. If we make the choice $\kappa_{n}=n$, at finite $N$ the regulated $w_{e}, v_{o}, T_{e o}, R_{o e}$ take the form $T_{e o}=T_{e o}^{(\infty)}\left(f_{e}^{N} / f_{o}^{N}\right)$, $R_{o e}=R_{o e}^{(\infty)}\left(f_{e}^{N} / f_{o}^{N}\right), \quad w_{e}=w_{e}^{(\infty)} f_{e}^{N}, \quad v_{o}=v_{o}^{(\infty)} / f_{o}^{N} \quad$ where $T^{(\infty)}$, $R^{(\infty)}, w^{(\infty)}, v^{(\infty)}$ are identical to the expressions in the infinite limit as in Eq. (4.3) but truncated as $N \times N$ matrices. The factors $f_{n}^{N}$ for $n=o, e$ are given by $f_{n}^{N}=\sqrt{\Gamma\left(N+\frac{1}{2}-\frac{1}{2} n\right) \Gamma\left(N+\frac{1}{2}+\frac{1}{2} n\right) / \Gamma\left(N+1-\frac{1}{2} n\right) \Gamma\left(N+1+\frac{1}{2} n\right)}$ $\times[\Gamma(N+1) / \Gamma(N+1 / 2)]$. This was computed by simply inserting $\kappa_{n}=n$ in the general regulated formulas for arbitrary $\kappa_{n}$ given in $[10,11,13]$. Note that the deformation factor $f_{n}^{N}$ stays pretty close to 1 (in the range 1.00-1.075) when $1 \leqslant n \leqslant N$, even for finite $N$, but grows as $n$ approaches $2 N$. For $n=2 N$, and large $N$, we get $f_{2 N}^{N}$ $\simeq(\pi N / 2)^{1 / 4}$. The strong deformation at the edges of the matrix can be avoided by using a different function $\kappa_{n}$ as a function of $n$. However, in our experience the simple choice $\kappa_{n}=n$ seems to work well in numerical computations even at small values of $N$. In the present example we see that it also works exactly at infinite $N$ in comparison to the continuous basis. 


$$
\begin{aligned}
\operatorname{det}(\bar{t} t)_{\text {discrete }} & =(1+\bar{w} w)_{\text {regulated }}^{-1 / 2}=\frac{\operatorname{det} \kappa_{o}}{\operatorname{det} \kappa_{e}} \\
& =\frac{1 \times 3 \times 5 \cdots(2 N-1)}{2 \times 4 \times 6 \cdots(2 N)} \\
& =\frac{\Gamma(2 N+1)}{2^{2 N} \Gamma^{2}(N+1)} \\
& \simeq(\pi N)^{-1 / 2}\left(1+\frac{1}{2 N}\right)^{-1 / 4}
\end{aligned}
$$

where the last expression is a good approximation for any $N \geqslant 1$ (including small $N$ ). Note both the leading $N$ behavior as well as the overall factor $(\pi / 2)^{-1 / 2}(2 N)^{-1 / 2}$, both of which are significant for the comparison of regulators in different bases. We compare this to the computation of $\operatorname{det}(\bar{t} t)_{k a p p a}$ in the kappa basis using $\rho(\kappa)$ as given in Eq. (4.24). We see that the leading term $1 /(2 \pi) \ln \left(2 N e^{\Delta}\right)$ in $\rho(\kappa)$ reproduces the correct $N$ dependence, while the second kappa dependent term produces precisely a factor of $\sqrt{2 / \pi}$, leading to the total result $\operatorname{det}(\bar{t} t)_{k a p p a}=\left(\pi N e^{\Delta}\right)^{-1 / 2}$. Comparing to the regulated discrete basis result in Eq. (5.62) we learn that we need $\Delta=0$ to get agreement between the two regulated results. Then, we seem to have an agreement between the computational procedures in the regulated discrete basis and the regulated kappa basis. However, we must warn the reader that $\Delta=0$ is still tentative because it is possible to change the regulator in the discrete basis, and we have not understood yet the principle that could fix it in either the discrete or the continuous basis. It is however significant that we have seen examples where $N$ as well as $\Delta$ cancel together in finite quantities at the critical dimension $d=26$. So, perhaps the value of $\Delta$ is not crucial as long as one is consistent in using the same regulator everywhere.

The third case is the exact computation of $\bar{v} v$ in the regulated discrete basis for any $N[10,11]$

$$
\begin{aligned}
(\bar{v} v)_{\text {discrete }} & =\left(\frac{\bar{w} w}{1+\bar{w} w}\right)_{\text {regulated }}=1-\frac{\operatorname{det} \kappa_{o}}{\operatorname{det} \kappa_{e}} \\
& =1-\frac{\Gamma(2 N+1)}{2^{2 N} \Gamma^{2}(N+1)} \underset{N \rightarrow \infty}{\rightarrow} 1 .
\end{aligned}
$$

At $N=\infty$ in the kappa basis we use $V_{o}(0)=\frac{1}{2} \sqrt{\pi o} v_{o}$ and obtain agreement with the discrete basis as follows

$$
(\bar{v} v)_{k a p p a}=\frac{8}{\pi} \lim _{\kappa \rightarrow 0}\left(\frac{1}{2} \bar{V}_{o} \frac{1}{o} V_{o}\right)_{\kappa \kappa}=\frac{8}{\pi}\left\langle 0\left|\frac{1}{L_{0}}\right| 0\right\rangle=1
$$

where we used Eq. (5.34).

In general we have noticed no problem in agreeing between the two bases for finite quantities where the effects of $N$ disappear. For such quantities it turns out that even a few modes in the discrete basis $(N \sim 5-10)$ gives very reliable numerical estimates of the exact $N=\infty$ values. However we advise care when the quantity blows up or vanishes as a power $N$ (such as determinants). Finite $N$ estimates are not predictably good for such quantities and furthermore they seem to be cutoff dependent (such as $\Delta$ ). When divergent quantities occur in combinations in which $N$ cancels, the various computational approaches seem to become reliable again, and furthermore in our experience, the result seems to be well estimated again at relatively small values of $N$.

\section{NONPERTURBATIVE LANDSCAPE}

The perturbative computations above have implications for nonperturbative computations in MSFT such as the ones in [14]. There a proposal was made for computing the true vacuum of Witten's string field theory at the classical level. This involved solving the equation of motion that follows from the action in Eq. (1.1)

$$
\left(L_{0}-1\right) A+\alpha^{\prime} g_{0} A \star A=0,
$$

for fields that are independent of the midpoint (hence the $\mathrm{D}_{25}$ brane vacuum). This could be done by treating the energy of the midpoint (called $\gamma$ ) as a perturbation and writing the rest of $L_{0}$ as a special star product form $\mathcal{L}_{0} \star A+A \star \mathcal{L}_{0}$. All solutions of Eq. (6.1), including the vacuum, were obtained exactly in the absence of $\gamma$. Then it was possible to setup a perturbation series in the midpoint energy $\gamma$ and compute analytically each term order by order. The lowest term was obtained as an exact solution $A^{(0)}\left(x_{e}, p_{e}\right)$, where $A^{(0)}\left(x_{e}, p_{e}\right)$ turned out to be related, up to an energy dependent factor $A^{(0)}=-\left(2 / \alpha^{\prime} g\right) \mathcal{L}_{0} \star P$, to the twisted butterfly projector $P=A_{b}\left(x_{e}, p_{e}\right)$ written in the MSFT basis. ${ }^{7}$ The next perturbation $A^{(1)}$ was also computed explicitly, while higher orders $A^{(n)}$ could be computed in a straightforward way with similar methods. Finally the energy of the vacuum and the tension of the $\mathrm{D}_{25}$ brane were given analytically up to second order in $\gamma$

$$
\begin{aligned}
T_{25} & =\frac{1}{V_{25}} S\left(A^{(0)}+\varepsilon A^{(1)}+\varepsilon^{2} A^{(2)}+\cdots\right) \\
& =\frac{-1}{\alpha^{\prime 3} g_{0}^{2}}\left(\frac{4}{3} \nu^{3}+\varepsilon \frac{\nu^{2} \delta}{2}\right)+O\left(\varepsilon^{2}\right),
\end{aligned}
$$

with

$$
\nu=\frac{1}{2}-\frac{d-2}{4}\left(\sum_{e>0} \kappa_{e}-\sum_{o>0} \kappa_{o}\right), \quad \delta=(d-2) \frac{\sum_{e>0} \kappa_{e} w_{e}^{2}}{1+\bar{w} w} .
$$

When this result was obtained the divergent nature of $\nu$ and $\delta$ were confusing. However the divergence of the bare

\footnotetext{
${ }^{7} \mathrm{~A}$ proposal based on the butterfly that has parallels to our program appeared recently [29].
} 
coupling constant $g_{0}$ was not noticed. We have seen in this paper in Eqs. (2.3), (4.32) that $g_{0}=g c\left(2 N e^{\Delta}\right)^{3 / 2}$. A quick look at $\nu, \delta$ show that they are both linearly divergent with $(2 N)$ as can be seen in a naive level truncation up to $(2 N)$ by inserting $\kappa_{o}=o, \kappa_{e}=e$ and using the unregulated $w_{e}$ $=-\sqrt{2}(-1)^{e / 2}$. Then the result for $T_{25}$ is perfectly finite at each order of the midpoint energy $\gamma$ since the factors of $(2 N)^{3}$ cancel between numerator and denominator in Eq. (6.3). Thus, this nonperturbative computation should proceed by using the same regulator consistently, keeping only the leading terms to order $(2 N)^{3}$ in the numerator and dropping everything nonleading. It reminds one of the large $N$ matrix computations. Progress on determining the vacuum state and energy using this approach will be reported in a future paper.

The observation above resolves another puzzle as follows. We recall the expression for $L_{0}$ that appears in the action of MSFT in Eq. (1.1). We display the version in [14] including ghosts $L_{0}=L_{0}^{\text {matter }}+L_{0}^{\text {ghost }}$,

$$
\begin{aligned}
L_{0}^{\text {matter }} & \sum_{e>0}\left(-\alpha^{\prime} \frac{\partial^{2}}{\partial x_{e}^{2}}-\frac{1}{16 \alpha^{\prime}} \kappa_{e}^{2} \frac{\partial^{2}}{\partial p_{e}^{2}}+\frac{1}{4 \alpha^{\prime}} \kappa_{e}^{2} x_{e}^{2}+4 \alpha^{\prime} p_{e}^{2}\right) \\
& +\frac{1}{2}(1+\bar{w} w) \beta_{0}^{2}+\frac{i \sqrt{2 \alpha^{\prime}}}{2} \beta_{0} \sum_{e>0} w_{e} \frac{\partial}{\partial x_{e}} \\
& -\frac{4 \alpha^{\prime}}{1+\bar{w} w}\left(\sum_{e>0} w_{e} p_{e}\right)^{2}-\frac{d}{2} \sum_{n=1}^{2 N} \kappa_{n}, \\
L_{0}^{g h o s t}= & i \sum_{e>0}\left(\frac{\partial}{\partial x_{e}^{b}} \frac{\partial}{\partial x_{e}^{c}}+\frac{1}{4} \kappa_{e}^{2} \frac{\partial}{\partial p_{e}^{b}} \frac{\partial}{\partial p_{e}^{c}}+\kappa_{e}^{2} x_{e}^{b} x_{e}^{c}+4 p_{e}^{b} p_{e}^{c}\right) \\
& -\frac{i}{1+\bar{w} w}\left(\sum_{e} w_{e} \frac{\partial}{\partial x_{e}^{b}}\right)\left(\sum_{e^{\prime}} w_{e^{\prime}} \frac{\partial}{\partial x_{e^{\prime}}^{c}}\right)+\sum_{n=1}^{2 N} \kappa_{n}
\end{aligned}
$$

where $\beta_{0}=-i \sqrt{2 \alpha^{\prime}}(\partial / \partial \bar{x})=-i \sqrt{2 \alpha^{\prime}}\left(\partial / \partial x_{c m}\right)$ represents the center of mass momentum. We had commented before in previous papers that the terms involving $(1+\bar{w} w)^{-1}$ are tricky. Even though they appear to vanish as $N \rightarrow \infty$ they actually contribute a finite term because infinite sums cancel the zero. The midpoint energy $\gamma$ mentioned above is in fact just the piece of $L_{0}$ proportional to $(1+\bar{w} w)^{-1}$.

But how about the divergent term in the first line $\frac{1}{2}(1$ $+\bar{w} w) \beta_{0}^{2}$ involving the center of mass momentum $\beta_{0}$ ? This divergence caused concern for some colleagues. Actually we can explain that this is the correct behavior of this term because otherwise there will be no center of mass momentum dependence in the large $N$ limit in nonperturbative physics. Let us start with the cubic term $g_{0} \operatorname{Tr}\left(A^{3}\right)$ and replace $A$ with $A=g_{0}^{-1} A^{\prime}$ so that it takes the form $g_{0}^{-2} \operatorname{Tr}\left(A^{\prime}\right)^{3}$. Doing the same to the quadratic term, we rewrite the action with an overall $g_{0}^{-2}$ as $S=-g_{0}^{-2}\left(\operatorname{Tr}\left[\left(1 / 2 \alpha^{\prime}\right) A^{\prime}\left(L_{0}-1\right) A^{\prime}\right.\right.$ $\left.\left.+(1 / 3) \operatorname{Tr}\left(A^{\prime}\right)^{3}\right]\right)$. Now it has the form of the computed nonperturbative energy in Eq. (6.3) and, from the discussion we gave above, we see that the numerator must behave like $N^{3}$ to compensate for the behavior of $g_{0}=\operatorname{cg}\left(2 N e^{\Delta}\right)^{3 / 2}$ in the denominator. It is clear that each $A^{\prime}$ accounts for a factor of $N$ in the numerator and also that $L_{0}$ contributes a factor of $N$ in the quadratic term. Now, if we also include momentum dependence (or $\bar{x}$ dependence) in $L_{0}$ the only way that momentum will not be negligible is by getting the help from the factor $(1+\bar{w} w) \sim 2 N$ in the form that it appears in $L_{0}$ $=\cdots+\frac{1}{2}(1+\bar{w} w) \beta_{0}^{2}$. This explains that this factor has precisely the correct behavior, and how it contributes in nonperturbative phenomena.

Of course, there is a way of eliminating the confusing factor by renormalizing $A^{\prime}=(1+\bar{w} w) \widetilde{A}$ and defining $\widetilde{L}_{0}$ $=(1+\bar{w} w)^{-1} L_{0}$, and further absorbing the extra factors in a definition $\widetilde{g}_{0}=(1+\bar{w} w)^{3 / 2} g_{0}$. Then in the new $\widetilde{L}_{0}$ every sum is divided by the factor $(1+\bar{w} w) \sim 2 N$ which is reminiscent of the finite quantities $\operatorname{Tr}(M) / N$ in large $N$ matrix theories. If the theory is redefined in this manner in terms of $\left(\widetilde{A}, \widetilde{L}_{0}\right)$ then every term of the action in every computation should be finite at every step, just like the leading terms in large $N$ matrix theories.

\section{CONCLUSION}

In this paper we have demonstrated that MSFT not only agreed in great detail with other computational approaches to Witten's string field theory, but it also led to new results that were not obtained before. We have developed several practical and theoretical tools on the way to the new results, and we have indicated how certain nonperturbative computations can be conducted by using the information provided in the present paper.

\section{ACKNOWLEDGMENTS}

I.B. would like to thank E. Fuchs, D. Gross, I. Kishimoto, M. Kroyter, Y. Matsuo, O. Oreshkov, S. Ramanujam, S. Samuel and W. Taylor for discussions, correspondence or comments. I.B. is in part supported by a DOE grant DEFG03-84ER40168. He is grateful to the CERN TH-division and to the KITP in Santa Barbara, for hospitality while this work was performed. I.P. is supported in part by DOE grant DE-FG02-91ER-40690, and he is grateful to S. D. Mathur for his support and encouragement.

\section{APPENDIX}

Anticipating future applications we compute also the following quantities by performing the integrals, which can be done easily by using an algebraic program. We give the results for five significant figures 


$$
\left(t \frac{2}{3+\bar{t} t} \kappa_{o}^{-1 / 2} v\right)_{e}=b_{e}=10^{-2} \times\left(\begin{array}{c}
22.222 \\
-11.058 \\
7.1982 \\
-5.2719 \\
4.1275 \\
\vdots
\end{array}\right), \quad(\mathrm{A} 1) \quad\left(\frac{2}{3+\bar{t} t} \kappa_{o}^{-1 / 2} v\right)_{o}=b_{o}=10^{-2} \times\left(\begin{array}{c}
54.433 \\
-12.804 \\
6.7119 \\
-4.4183 \\
3.2432 \\
\vdots
\end{array}\right) \text {, }
$$

$$
(\bar{t} t)_{o o^{\prime}}=10^{-2} \times\left(\begin{array}{cccccc}
40.528 & 23.399 & -14.097 & 9.8718 & -7.4881 & \ldots \\
23.399 & 85.56 & 10.464 & -8.1365 & 6.6112 & \ldots \\
-14.097 & 10.464 & 91.684 & 6.8506 & -5.795 & \ldots \\
9.8718 & -8.1365 & 6.8506 & 94.133 & 5.1061 & \ldots \\
-7.4881 & 6.6112 & -5.795 & 5.1061 & 95.46 & \ldots \\
\vdots & \vdots & \vdots & \vdots & \vdots & \ddots
\end{array}\right)
$$

Note the increasing diagonal, although the off-diagonals are much smaller. The increase on the diagonal $(\bar{t} t)_{o o}$ quickly slows down as $o$ increases, and stays around $160<(\bar{t} t)_{o o}<167$ in the range $100<o<175$ and then makes a sharp drop reaching $(\bar{t} t)_{o o}=2.2614$ at $o=185$, and continues to drop slowly as $o$ increases.

The following are exact computations, like the ones above, obtained directly by using the integrals, not by inserting a truncated form of $t \bar{t}$ or $\bar{t} t$. If compared to what follows from the truncated $t \bar{t}$ or $\bar{t} t$ one finds results that disagree although they are in a similar range of values. Therefore, the contributions from the higher modes are not always negligible.

$$
\begin{gathered}
\left(\frac{1-t \bar{t}}{3+t \bar{t}}\right)_{e e^{\prime}}=M_{e e^{\prime}}=10^{-2} \times\left(\begin{array}{cccccc}
5.3498 & -3.6787 & 2.7533 & -2.1837 & 1.8013 & \ldots \\
-3.6787 & 2.8931 & -2.3337 & 1.9422 & -1.6571 & \ldots \\
2.7533 & -2.3337 & 1.9739 & -1.6978 & 1.4843 & \ldots \\
-2.1837 & 1.9422 & -1.6978 & 1.4961 & -1.3323 & \ldots \\
1.8013 & -1.6571 & 1.4843 & -1.3323 & 1.2039 & \ldots \\
\vdots & \vdots & \vdots & \vdots & \vdots & \ddots
\end{array}\right) \\
\left(\frac{1-\bar{t} t}{3+\bar{t} t}\right)_{o o^{\prime}}=M_{o o^{\prime}}=10^{-2} \times\left(\begin{array}{cccccc}
18.519 & -7.6030 & 4.7259 & -3.3933 & 2.6287 & \ldots \\
-7.6030 & 4.5369 & -3.2882 & 2.5723 & -2.106 & \ldots \\
4.7259 & -3.2882 & 2.5820 & -2.1243 & 1.8012 & \ldots \\
-3.3933 & 2.5723 & -2.1243 & 1.8088 & -1.5725 & \ldots \\
2.6287 & -2.106 & 1.8012 & -1.5725 & 1.3933 & \ldots \\
\vdots & \vdots & \vdots & \vdots & \vdots & \ddots
\end{array}\right) \\
\left(\frac{t \bar{t}-1}{1+3 t \bar{t}}\right)_{e e^{\prime}}=\tilde{M}_{e e^{\prime}}=10^{-2} \times\left(\begin{array}{cccccc}
7.8189 & -5.7480 & 4.5367 & -3.7613 & 3.2233 & \ldots \\
-5.7480 & 4.6577 & -3.8725 & 3.3154 & -2.9035 & \ldots \\
4.5367 & -3.8725 & 3.3266 & -2.9122 & 2.5918 & \ldots \\
-3.7613 & 3.3154 & -2.9122 & 2.5913 & -2.3348 & \ldots \\
3.2233 & -2.9035 & 2.5918 & -2.3348 & 2.1241 & \ldots \\
\vdots & \vdots & \vdots & \vdots & \vdots & \ddots
\end{array}\right)
\end{gathered}
$$




$$
\left.(\bar{t} \overline{1+1})_{o \bar{t}^{\prime}}\right)_{o o^{\prime}}=\widetilde{M}_{o o^{\prime}}=10^{-2} \times\left(\begin{array}{cccccc}
40.741 & 19.007 & -12.905 & 9.9171 & -8.1191 & \ldots \\
19.007 & 10.664 & 7.8008 & -6.2376 & 5.2334 & \ldots \\
-12.905 & 3.2882 & 5.9573 & 4.8957 & -4.1851 & \ldots \\
9.9171 & -6.2376 & 4.8957 & 4.1014 & 3.5561 & \ldots \\
-8.1191 & 5.2334 & -4.1851 & 3.5561 & 3.1171 & \ldots \\
\vdots & \vdots & \vdots & \vdots & \vdots & \ldots
\end{array}\right) .
$$

Other matrix elements of interest are obtained from the ones above, such as $4 /(3+t \bar{t})=1+M$, and $2(1+t \bar{t}) /(3+t \bar{t})$ $=1-M$, etc.

We can also evaluate some of the quantities by using other methods and compare to the results above. In particular, by exact summation over the infinite modes we can express the infinite matrices $(t \bar{t})_{e e^{\prime}}$ and $(\bar{t} t)_{o o^{\prime}}$ in terms of the generalized hypergeometric function as follows:

$$
\begin{aligned}
(\bar{t} t)_{o o^{\prime}}= & \frac{32(i)^{o-o^{\prime}} \sqrt{o o^{\prime}}}{\pi^{2}\left(o^{2}-4\right)\left(\left(o^{\prime}\right)^{2}-4\right)} \\
& \times \text { hypergeom }\left(\left[2,1+\frac{o}{2}, 1-\frac{o^{\prime}}{2}, 1+\frac{o^{\prime}}{2}, 1-\frac{o}{2}\right],\right. \\
& {\left.\left[2+\frac{o}{2}, 2-\frac{o}{2}, 2-\frac{o^{\prime}}{2}, 2+\frac{o^{\prime}}{2}\right], 1\right) . }
\end{aligned}
$$

The values of the hypergeometric function exactly agree with the results of the integrals given above.

Also we recognize the quantities $\omega, b_{o}, b_{e}, M_{o o^{\prime}}, M_{e e^{\prime}}, \widetilde{M}_{o o^{\prime}}, \widetilde{M}_{e e^{\prime}}$ from our earlier work on the computation of the Neumann matrices by using the Moyal product $[11,15]$ and comparing to Neumann coefficients which were obtained from conformal field theory [7]. Therefore, in some sense we already knew the result for these quantities. However, what we knew is only a special case of the more general formulas given in Eqs. (4.8)-(4.13), and serves to confirm the general method. For example

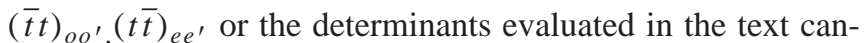
not be obtained by using just the Neumann matrices. Thus, from our earlier work we extract the following results given in terms of the vectors $A_{e}, A_{o}, B_{e}, B_{o}$ whose numerical values are given by the following generating functions:

$$
\begin{aligned}
& \left(\frac{1+z}{1-z}\right)^{1 / 3}=1+\sum A_{e} z^{e}+i \sum A_{o} z^{o}, \\
& \left(\frac{1+i z}{1-i z}\right)^{2 / 3}=1+\sum B_{e} z^{e}+i \sum B_{o} z^{o} .
\end{aligned}
$$

In terms of these we have the desired quantities as obtained from $[11,15]$ with a little algebra

$$
\begin{aligned}
& b_{o}=\left(\frac{2}{3+\bar{t} t} \kappa_{o}^{-1 / 2} v\right)_{o}=\sqrt{\frac{2}{3}} \frac{A_{o}}{\sqrt{o}}, \\
& \widetilde{b}_{o}=\left(\frac{2}{1+3 \bar{t} t} \kappa_{o}^{-1 / 2} v\right)_{o}=\sqrt{\frac{2}{3}} \frac{B_{o}}{\sqrt{o}} \\
& b_{e}=\left(t \frac{2}{3+\bar{t} t} \kappa_{o}^{-1 / 2} v\right)_{e}=-\sqrt{2} \frac{A_{e}}{\sqrt{e}}, \\
& \widetilde{b}_{e}=\left(t \frac{2}{1+3 \bar{t} t} \kappa_{o}^{-1 / 2} v\right)_{e}=-\frac{\sqrt{2}}{3} \frac{B_{e}}{\sqrt{e}}
\end{aligned}
$$

and

$$
\begin{aligned}
& M_{o o^{\prime}}=\left(\frac{1-\bar{t} t}{3+\bar{t} t}\right)_{o o^{\prime}}= \begin{cases}\frac{1}{3}\left(1-A_{o}^{2}-2 \sum_{o^{\prime \prime}=1}^{o-2} A_{o^{\prime \prime}}^{2}+2 \sum_{e^{\prime \prime}=2}^{o-1} A_{e^{\prime \prime}}^{2}\right), & \text { for } o=o^{\prime}, \\
\frac{1}{3} \sqrt{o o^{\prime}}\left(\frac{(A \bar{B}+B \bar{A})_{o o^{\prime}}}{o+o^{\prime}}+\frac{(A \bar{B}-B \bar{A})_{o o^{\prime}}}{o-o^{\prime}}\right), & \text { for } o \neq o^{\prime},\end{cases} \\
& M_{e e^{\prime}}=\left(\frac{1-t \bar{t}}{3+t \bar{t}}\right)_{e e^{\prime}}= \begin{cases}\frac{1}{3}\left(1+A_{e}^{2}+2 \sum_{e^{\prime \prime}=2}^{e-2} A_{e^{\prime \prime}}^{2}-2 \sum_{o^{\prime \prime}=1}^{e-1} A_{o^{\prime \prime}}^{2}\right), & \text { for } e=e^{\prime}, \\
\frac{1}{3} \sqrt{e e^{\prime}}\left(\frac{(A \bar{B}+B \bar{A})_{e e^{\prime}}}{e+e^{\prime}}+\frac{(A \bar{B}-B \bar{A})_{e e^{\prime}}}{e-e^{\prime}}\right), & \text { for } e \neq e^{\prime},\end{cases}
\end{aligned}
$$




$$
Y_{e o}=\left(t \frac{1}{3+t \bar{t}}\right)_{e o}=-\frac{\sqrt{e o}}{4 \sqrt{3}}\left(\frac{(A \bar{B}-B \bar{A})_{e o}}{e+o}+\frac{(A \bar{B}+B \bar{A})_{e o}}{e-o}\right)
$$

where a bar on a vector, such as $\bar{A}$, means its transpose. Similarly we have

$$
\begin{aligned}
& \tilde{M}_{o o^{\prime}}=\left(\frac{1-\bar{t} t}{1+3 \bar{t} t}\right)_{o o^{\prime}}=\left\{\begin{array}{l}
-\frac{1}{3}\left(1-A_{o}^{2}-2 \sum_{o^{\prime \prime}=1}^{o-2} A_{o^{\prime \prime}}^{2}+2 \sum_{e^{\prime \prime}=2}^{o-1} A_{e^{\prime \prime}}^{2}-2 A_{o} B_{o}\right), \quad \text { for } o=o^{\prime}, \\
-\frac{1}{3} \sqrt{o o^{\prime}}\left(\frac{(A \bar{B}+B \bar{A})_{o o^{\prime}}}{o+o^{\prime}}-\frac{(A \bar{B}-B \bar{A})_{o o^{\prime}}}{o-o^{\prime}}\right), \text { for } o \neq o^{\prime},
\end{array}\right. \\
& \tilde{M}_{e e^{\prime}}=\left(\frac{1-\bar{t} t}{1+3 t \bar{t}}\right)_{e e^{\prime}}=\left\{\begin{array}{l}
-\frac{1}{3}\left(1+A_{e}^{2}+2 \sum_{e^{\prime \prime}=2}^{e-2} A_{e^{\prime \prime}}^{2}-2 \sum_{o^{\prime \prime}=1}^{e-1} A_{o^{\prime \prime}}^{2}-2 A_{e} B_{e}\right), \quad \text { for } e=e^{\prime}, \\
+\frac{1}{3} \sqrt{e e^{\prime}}\left(\frac{(A \bar{B}+B \bar{A})_{e e^{\prime}}}{e+e^{\prime}}-\frac{(A \bar{B}-B \bar{A})_{e e^{\prime}}}{e-e^{\prime}}\right),
\end{array}\right. \\
& \tilde{Y}_{e o}=\left(t \frac{1}{1+3 t \bar{t}}\right)_{e o}=\frac{\sqrt{e o}}{4 \sqrt{3}}\left(\frac{(A \bar{B}-B \bar{A})_{e o}}{e+o}-\frac{(A \bar{B}+B \bar{A})_{e o}}{e-o}\right) .
\end{aligned}
$$

The generating functions give the following values for $A_{e}, A_{o}, B_{e}, B_{o}$ which are useful in the present paper:

$$
\begin{aligned}
& \sum A_{e} z^{e}=-\frac{2}{3^{2}} z^{2}+\frac{2 \times 19}{3^{5}} z^{4}-\frac{2 \times 409}{3^{8}} z^{6}+\frac{2 \times 11 \times 283}{3^{10}} z^{8}-\frac{2 \times 220721}{3^{14}} z^{10}+\ldots \\
& \sum A_{o} z^{o}=\frac{2}{3} z-\frac{2 \times 11}{3^{4}} z^{3}+\frac{2 \times 67}{3^{6}} z^{5}-\frac{2 \times 1409}{3^{9}} z^{7}+\frac{2 \times 94993}{3^{13}} z^{9}+\cdots \\
& \sum B_{e} z^{e}=-\frac{2^{3}}{3^{2}} z^{2}+\frac{2^{4} 11}{3^{5}} z^{4}-\frac{2^{3} 523}{3^{8}} z^{6}+\frac{2^{5} 29 \times 37}{3^{10}} z^{8}-\frac{2^{3} 323381}{3^{14}} z^{10}+\ldots \\
& \sum B_{o} z^{o}=\frac{2^{2}}{3} z-\frac{2^{2} 17}{3^{4}} z^{3}+\frac{2^{2} 127}{3^{6}} z^{5}-\frac{2^{2} 11 \times 277}{3^{9}} z^{7}+\frac{2^{2} 23 \times 9839}{3^{13}} z^{9}+\cdots
\end{aligned}
$$

By applying these formulas we verified that the expressions in terms of the vectors $A_{n}, B_{n}$ given in Eqs. (A10)-(A17) agree with the results produced by the integrals in Eqs. (4.8)-(4.13) as listed in Eqs. (A1)-(A7).

[1] E. Witten, Nucl. Phys. B268, 253 (1986).

[2] S. Samuel, Nucl. Phys. B308, 285 (1988).

[3] J.H. Sloan, Nucl. Phys. B302, 349 (1988).

[4] S. Mandelstam, Phys. Rep. 13C, 259 (1974).

[5] S. Giddings, Nucl. Phys. B278, 242 (1986).

[6] E. Cremmer, A. Schwimmer, and C. Thorn, Phys. Lett. B 179, 307 (1986).

[7] D. Gross and A. Jevicki, Nucl. Phys. B283, 1 (1987); B287, 225 (1987).

[8] N. Ohta, Phys. Rev. D 34, 3785 (1986); 35, 2627(E) (1987).

[9] I. Bars, Phys. Lett. B 517, 436 (2001).
[10] I. Bars and Y. Matsuo, Phys. Rev. D 65, 126006 (2002).

[11] I. Bars and Y. Matsuo, Phys. Rev. D 66, 066003 (2002).

[12] I. Bars, hep-th/0211238.

[13] I. Bars, I. Kishimoto, and Y. Matsuo, Phys. Rev. D 67, 066002 (2003).

[14] I. Bars, I. Kishimoto, and Y. Matsuo, Phys. Rev. D 67, 126007 (2003).

[15] I. Bars, I. Kishimoto, and Y. Matsuo, J. High Energy Phys. 07, 027 (2003).

[16] M.R. Douglas, H. Liu, G. Moore, and B. Zwiebach, J. High Energy Phys. 04, 022 (2002). 
[17] T.G. Erler, hep-th/0205107.

[18] V.A. Kostelecky and R. Potting, Phys. Rev. D 63, 046007 (2001).

[19] W. Taylor, hep-th/0207132, and references therein.

[20] A. Le Clair, E. Peskin, and C.R. Preitschopf, Nucl. Phys. B317, 411 (1989); B317, 464 (1989).

[21] L. Rastelli, A. Sen, and B. Zwiebach, J. High Energy Phys. 03, 029 (2002).

[22] I. Bars, Lecture Notes on Quantum Mechanics, http:// physics.usc.edu/ bars/courses/book2002.pdf (link from home page, under "teaching").

[23] Abramovitz and Stegun, formula (15.3.13) taken for large $z$.

[24] D.M. Belov and C. Lovelace, Phys. Rev. D 68, 066003 (2003).

[25] D.M. Belov and A. Konechny, Phys. Lett. B 558, 111 (2003); J. High Energy Phys. 10, 049 (2002).

[26] E. Fuchs, M. Kroyter, and A. Marcus, J. High Energy Phys. 11, 046 (2202); 11, 039 (2003).

[27] D.M. Belov, hep-th/0210199.

[28] T.G. Erler, hep-th/0304044.

[29] Y. Okawa, Phys. Rev. D 69, 086001 (2004). 